\title{
Cell fate decisions within the mouse organizer are governed by graded Nodal signals
}

\author{
Stéphane D. Vincent, N. Ray Dunn, Shigemi Hayashi, Dominic P. Norris, ${ }^{1}$ \\ and Elizabeth J. Robertson ${ }^{2}$ \\ Department of Molecular and Cellular Biology, Harvard University, Cambridge, Massachusetts 02138, USA
}

\begin{abstract}
It is well known that cell fate decisions in the mouse organizer region during gastrulation ultimately govern gut formation and patterning, left-right axis determination, and development of the central nervous system. Previous studies suggest that signaling pathways activated by Nodal, bone morphogenetic protein (BMP), and Wnt ligands coordinately regulate patterning of the streak and the formation of midline organizing tissues, but the specific contributions of these molecules within discrete cell lineages are poorly defined. Here we removed Smad2 activity in the epiblast, using a conditional inactivation strategy. Abrogation of Smad2 does not compromise primitive streak (PS) formation or gastrulation movements, but rather results in a failure to correctly specify the anterior definitive endoderm (ADE) and prechordal plate (PCP) progenitors. To selectively lower Nodal activity in the posterior epiblast, we generated a novel allele lacking the proximal epiblast enhancer (PEE) governing Nodal expression in the PS. As for conditional inactivation of Smad2, germ-line deletion of the PEE selectively disrupts development of the anterior streak. In striking contrast, the node and its midline derivatives, the notochord and floor plate, develop normally in both categories of mutant embryos. Finally, we show that removal of one copy of Smad3 in the context of a Smad2-deficient epiblast results in a failure to specify all axial midline tissues. These findings conclusively demonstrate that graded Nodal/Smad 2 signals govern allocation of the axial mesendoderm precursors that selectively give rise to the ADE and PCP mesoderm.
\end{abstract}

[Keywords: Nodal; Smad2; organizer; mouse embryo; axis patterning]

Received April 3, 2003; revised version accepted May 9, 2003.

Shortly after implantation, reciprocal signaling between the three cell populations of the early mouse embryo, namely, the epiblast, primitive visceral endoderm (VE), and extraembryonic ectoderm, establishes the initial proximal-distal (P-D) axis. The anterior-posterior (A-P) axis emerges gradually as a result of subsequent cell movements, and first becomes evident when a discrete population of proximal epiblast cells is induced to form mesoderm, initiating primitive streak (PS) formation and marking the posterior side of the embryo (for reviews, see Beddington and Robertson 1999; Lu et al. 2001). Over the next 12-24 h, the PS gradually elongates to reach the distal tip of the epiblast. Fate mapping studies have shown that cells entering the streak proximally give rise to extraembryonic mesoderm. The lateral plate and paraxial mesoderm emerge from intermediate levels (for review, see Lawson 1999), whereas cells situated in the anterior streak give rise to the so termed "organizer re-

${ }^{1}$ Present address: MRC Mammalian Genetics Unit, Oxfordshire, OX11 ORD United Kingdom.

${ }^{2}$ Corresponding author.

E-MAIL ejrobert@fas.harvard.edu; FAX (617) 496-6770.

Article and publication are at http://www.genesdev.org/cgi/doi/10.1101/ gad.1100503. gion". First identifiable at midstreak stages by the expression of the transcription factors Gsc and Foxa2 (for review, see Camus and Tam 1999|, these most anterior cells include "axial mesendoderm" precursors that give rise to the anterior definitive endoderm (ADE) and prechordal plate (PCP) mesoderm, as well as progenitors of the node and its derivatives, the notochord and floor plate. These early cell fate decisions during gastrulation ultimately govern gut formation and patterning, leftright (L-R) axis determination, and development of the central nervous system (CNS).

In the mouse, initial anterior identity is imposed by a population of specialized VE cells, termed the anterior visceral endoderm (AVE), that overlies the prospective anterior side of the epiblast. Previous studies have shown that the TGF $\beta$ (transforming growth factor $\beta$ ) family member Nodal acts from the epiblast to activate the intracellular effector molecule Smad2 in the VE and to promote formation of the AVE (Brennan et al. 2001). Failure to form the AVE results in a complete loss of anterior markers in the epiblast (Waldrip et al. 1998; Perea-Gomez et al. 2002). As gastrulation proceeds, the AVE is displaced proximally (Thomas and Beddington 1996), and patterning of the neurectoderm is assumed by 
the anterior streak derivatives including the anteriormost definitive endoderm, PCP and notochord. Genetic studies provide limited insight into the developmental relationships among these specific cell types. For example, loss of the transcription factor $\mathrm{Hex}$, expressed in the ADE, disrupts forebrain patterning (Martinez Barbera et al. 2000), whereas loss of Lhx1 activity in the PCP results in severe anterior truncations (Shawlot et al. 1999|. Interestingly, the node is not required for initial $\mathrm{A}-\mathrm{P}$ axis specification per se, because the neural tube retains rudimentary patterning along the A-P axis after physical or genetic removal of the node (Dufort et al. 1998; Davidson et al. 1999; Klingensmith et al. 1999). Collectively, these findings demonstrate that derivatives of the anterior streak precisely pattern the overlying neurectoderm, yet their discrete functional activities are poorly understood.

The induction and patterning of the anterior streak requires the activities of two fork head transcription factors, Foxa2 and FoxH1 (Ang and Rossant 1994; Weinstein et al. 1994; Hoodless et al. 2001; Yamamoto et al. 2001). Foxa2 is broadly expressed throughout the VE and locally in anterior streak cells, where it is required to form the node (Ang and Rossant 1994; Weinstein et al. 1994; Dufort et al. 1998). FoxH1 is expressed throughout the epiblast and VE (Weisberg et al. 1998; Saijoh et al. 2000) and collaborates with phosphorylated Smad2/3/4 complexes to regulate targets of the Nodal pathway (for review, see Whitman 2001). FoxH1 promotes Foxa2 expression levels in the anterior streak and consequently anterior streak derivatives are missing in FoxH1 mutant embryos (Hoodless et al. 2001; Yamamoto et al. 2001). Thus, it appears likely that FoxH1 transduces Nodal signals responsible for patterning the anterior streak and establishing of the mouse organizer region. The Nodal antagonists Leftyl and Cerl are necessary for correct positioning and patterning of PS tissues (Perea-Gomez et al. 2002). In contrast, the bone morphogenetic protein (BMP) antagonists Noggin and Chordin are required later for axial mesendoderm formation (Bachiller et al. 2000), whereas specification of the anterior neurectoderm depends on expression of the Wnt antagonist Dkk1 in the anterior mesendoderm (Mukhopadhyay et al. 2001). Thus studies of induced mutations suggest that signaling pathways activated by Nodal, BMP, and Wnt ligands coordinately regulate patterning of the streak and the formation of midline organizing tissues, but the specific activities of these molecules during cell type specification have not been elucidated.

Nodal signaling via the Alk4 or Alk7 type I receptors in association with either the ActRIIA or ActRIIB type II receptors activates the intracellular effectors Smad2 and Smad3, which in turn associate with Smad4 and translocate to the nucleus to regulate target gene expression (for reviews, see Massagué et al. 2000; Whitman 2001). Smad2 signals contributed by the extraembryonic tissues are essential for establishing the AVE and hence A-P identity within the epiblast (Waldrip et al. 1998). In the absence of Smad2, the entire epiblast adopts an extraembryonic mesodermal fate, giving rise to a normal yolk sac and fetal blood cells (Waldrip et al. 1998; Heyer et al. 1999). In chimeric embryos, Smad2 mutant cells extensively colonize ectodermal and mesodermal populations without disturbing normal development but are not recruited into the definitive endoderm (DE) lineage during gastrulation (Tremblay et al. 2000). Thus, it seems likely that the closely related effector Smad3 acts downstream of essential Nodal signals during gastrulation and mesodermal patterning. However, defects observed in chimeric embryos potentially reflect the developmental bias of embryonic stem (ES) cells during tissue colonization as opposed to functional contributions of the Nodal/Smad2 pathway during cell fate decisions.

Here we remove Smad2 activity in the epiblast using a conditional gene inactivation strategy. In the absence of Smad2, PS formation and gastrulation proceed normally, but correct specification of the ADE and PCP progenitors fails. To selectively lower Nodal activity in the posterior epiblast, we generated a novel Nodal allele lacking the proximal epiblast enhancer (PEE) governing expression in the proximal epiblast (Norris and Robertson 1999). As for conditional inactivation of Smad2, germ line deletion of the PEE selectively disrupts development of the anterior streak. In striking contrast, the node and its midline derivatives, the notochord and floor plate, develop normally in both categories of mutant embryos. Finally, we show that removal of one copy of Smad3 in the context of a Smad2-deficient epiblast results in a failure to specify all axial midline tissues. Thus, we conclude that cell fate decisions within the anterior streak are governed by graded Nodal/Smad2 signals, and that Smad2dependent signals regulate formation of the definitive endoderm and PCP precursor populations.

\section{Results}

Generation of a Smad2 conditional allele

Our previous studies have shown that Smad2 is uniquely required in the VE, where it functions in the pathway that specifies the AVE (Waldrip et al. 1998; Brennan et al. 2001). Accordingly, in Smad2-deficient embryos the epiblast forms extensive extraembryonic mesoderm (Waldrip et al. 1998; Heyer et al. 1999; Brennan et al. 2001). Smad2-deficient ES cells efficiently give rise to embryonic mesoderm and ectoderm, as well as the node and its derivatives, but rarely contribute to the DE (Tremblay et al. 2000). To further evaluate Smad2 functions during specification of these discrete cell lineages during gastrulation, we designed a strategy to delete Smad2 activity from the epiblast prior to gastrulation.

We generated a conditional Smad2 allele (Smad2 ${ }^{\text {RobCA }}$ designated Smad2 ${ }^{\mathrm{CA}}$ ) by flanking the first coding exon with loxP sites (Fig. 1A,B). Smad2 ${ }^{\mathrm{CA} / \mathrm{CA}}$ animals are viable and fertile (Fig. 1C), and the locus efficiently undergoes Cre-mediated recombination in tail cartilage tissue from animals harboring a Col2a1Cre transgene (Fig. 1D; Ovchinnikov et al. 2000). To confirm that the remaining loxP sites do not compromise transcriptional activity, 
Vincent et al.
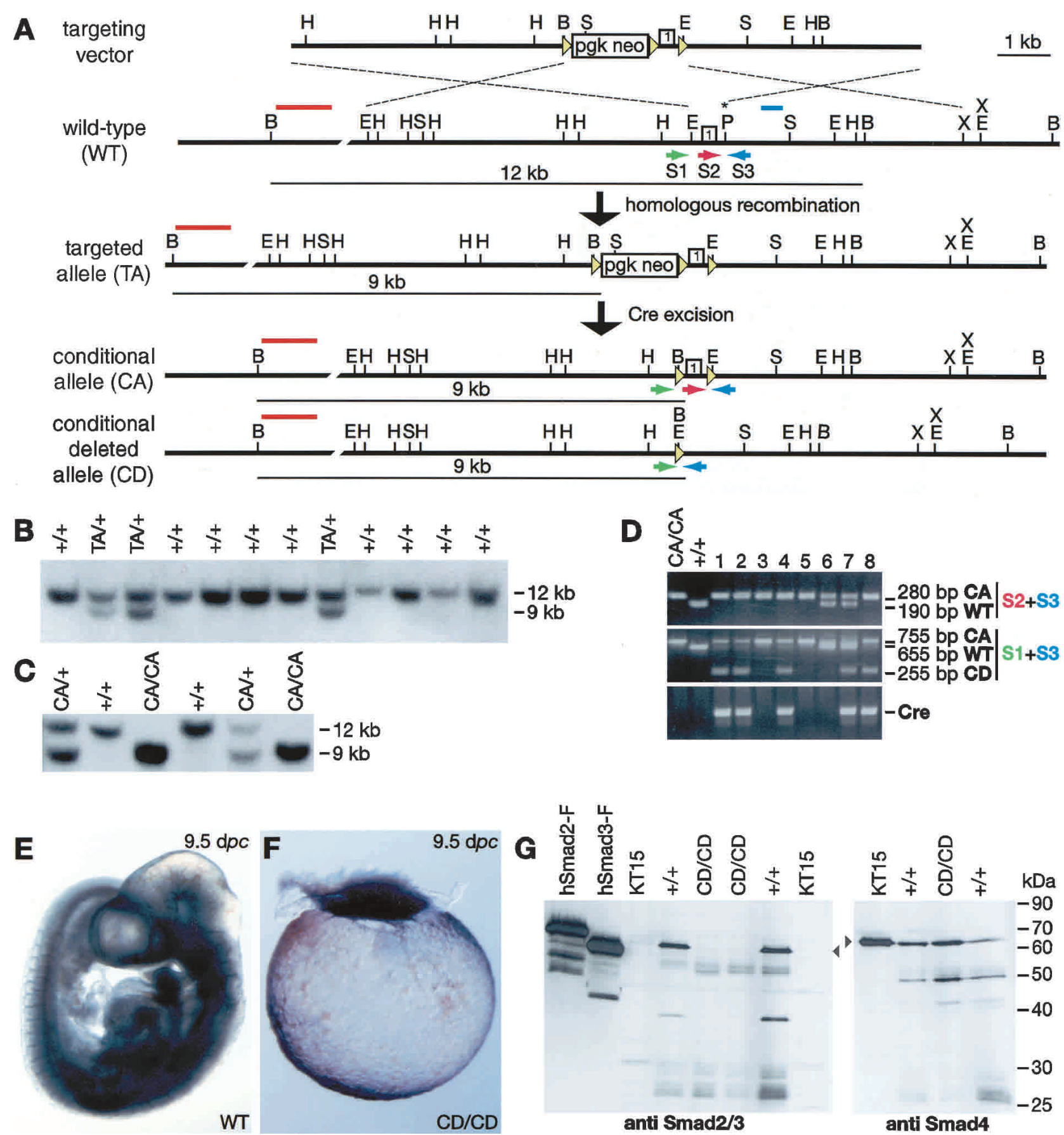

Figure 1. Generation and characterization of a Smad2 conditional allele. (A) Strategy used to flank the first coding exon of Smad2 with loxP sites. Targeted clones were identified using a 5' external probe (red line) and an internal probe (blue line). Primers used for PCR are indicated by arrows. B, BamHI; E, EcoRI; H, HindIII; P, PstI (the asterisk indicates that a loxP site was introduced at this position); S, SpeI; X, XhoI. (B) Southern blot analysis of drug-resistant ES cell clones digested with BamHI and screened using the 5' probe yields wild-type (WT; 12-kb) and targeted $(9-\mathrm{kb})$ alleles. $(C)$ Southern blot analysis of BamHI-digested tail DNAs from a Smad2 ${ }^{\mathrm{CA} /+}$ intercross litter. Smad2 ${ }^{\mathrm{CA} / \mathrm{CA}}$ animals are born at Mendelian ratios and are viable and fertile. $(D)$ PCR analysis of tail-tip DNA samples from offspring from matings between Smad2 ${ }^{\mathrm{CA} / \mathrm{CA}}$ and Col2a1Cre/+ animals that express Cre in the cartilage (Ovchinnikov et al. 2000). Animals 1, 2, 4, 7, and 8 carry the Cre transgene and the Smad2CD allele. (E) A 9.5-dpc wild-type (WT) embryo. (F) A 9.5-dpc Smad2 $\mathrm{CD} / \mathrm{CD}$ embryo composed only of extraembryonic tissues phenocopies Smad2 ${ }^{\text {Robm1/Robm1 }}$ embryos (Waldrip et al. 1998). (G) Western blot analysis with a monoclonal Smad2 antibody (left blot) and, as a control, a monoclonal Smad4 antibody (right blot). Smad2 and Smad3 are expressed by COS cells transfected with Smad2-Flag (hSmad2-F) or Smad3-Flag (hSmad3-F) expression constructs and 9.5-dpc wild-type (WT) yolk sacs, but no Smad2 signal was detected in Smad2 ${ }^{\text {Robm1/Robm1 }}$ KT15 ES cells or Smad2 ${ }^{\mathrm{CD} / \mathrm{CD}}$ embryo lysates. The positions of Smad2 $(58 \mathrm{kD})$ and Smad4 $(66 \mathrm{kD})$ are indicated by arrowheads. 
we crossed the Smad2 ${ }^{\mathrm{CA}}$ allele into the $S m a d 2^{\mathrm{Robm} 1}$ null background (Waldrip et al. 1998) and obtained viable
Smad2 ${ }^{\mathrm{CA} / \text { Robm1 }}$ progeny at the expected Mendelian ratio (data not shown). Moreover, germ line excision
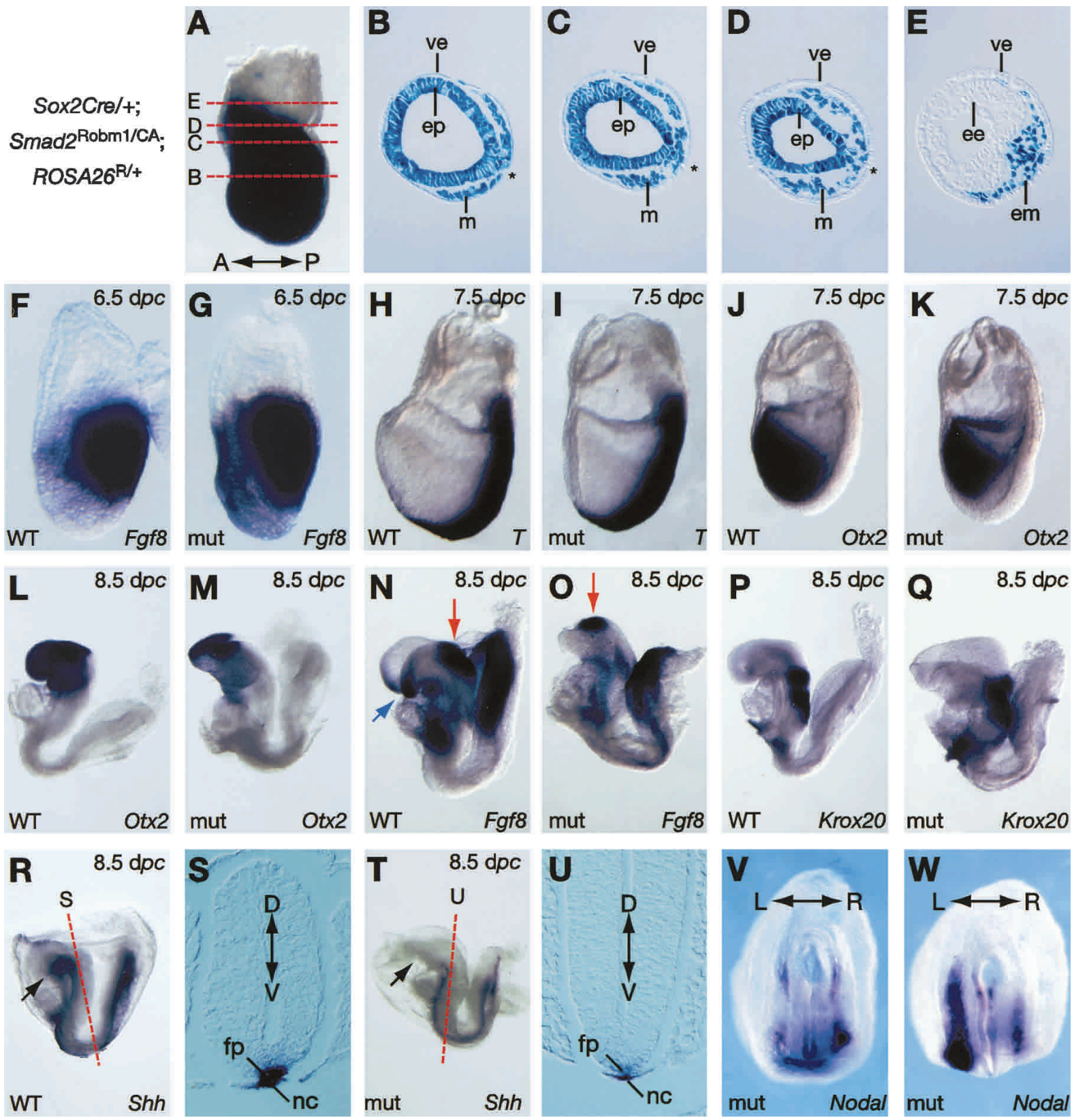

Figure 2. Deletion of Smad2 from the epiblast does not perturb gastrulation but leads to anterior patterning defects. (A) LacZ expression in 7.5-dpc Sox $2 \mathrm{Cre} /+;$ Smad2 ${ }^{\mathrm{CA} / \mathrm{Robm} 1}{ }_{;} R_{\mathrm{OSA}} 26^{\mathrm{R} /+}$ embryo. $(B-E)$ Sections of the embryo indicated in $A$. The extraembryonic ectoderm and visceral endoderm (VE) fail to express $L a c Z$, whereas the epiblast and mesoderm are uniformly blue. ve, visceral endoderm; ep, epiblast; $\mathrm{m}$, mesoderm; ee, extraembryonic ectoderm. The asterisk indicates the primitive streak. $(F-W)$ Whole-mount in situ hybridization of control $(F, H, J, L, N, P, R)$ and Sox2Cre/+;Smad2 CA/Robm1 mutants $(G, I, K, M, O, Q, U)$. $(F-I)$ Streak formation and elongation proceed normally, as shown by Fgf8 and T expression. $(J, K)$ Otx2, marker of the neural plate, is correctly expressed at 7.5 dpc. At early somite stages, the loss of anterior head structures is revealed by reduced expression of Otx2 $(L, M)$ and by loss of the anterior neural ridge (blue arrow in $N$ ) corresponding to the most anterior Fgf8 domain $(N, O)$. However, Fgf8 expression at the $\mathrm{mid} /$ hindbrain boundary (red arrow) is retained $(N, O)$, and hindbrain formation is normal as assessed by Krox20 expression in rhombomeres 3 and $5(P, Q) .(R, T)$ Anterior truncation is also shown by the loss of the ventral domain of $S h h$ (black arrow) in the brain. $(S, U)$ In contrast, other Shh expression domains including the notochord (nc) and the floor plate (fp) are unaffected, as shown in frontal sections. $(V, W)$ Nodal, normally confined to the left lateral plate mesoderm at the 3-5 somite stage, is bilaterally expressed in some mutant embryos. $(A-R, T)$ Lateral views with anterior to the left. $(V, W)$ Ventral views. A-P, anterior-posterior axis; D-V, dorsal-ventral axis; L-R, left-right axis. 
of the loxP flanked exon using a Prm1-Cre deleter strain (O'Gorman et al. 1997) generates a null allele: Smad2 $2^{\mathrm{CD} / \mathrm{CD}}$ or Smad2 $\mathrm{CD} /$ Robm1 embryos phenocopy Smad2 ${ }^{\text {Robml }}$ null mutants (Fig. 1F). Finally, Western blot analysis confirms that Smad2 $\mathrm{CD} / \mathrm{CD}$ embryos lack detectable Smad2 protein (Fig. 1G).

Inactivation of Smad2 in the epiblast selectively disturbs development of anterior streak derivatives

For conditional inactivation of Smad2, we used a Sox2Cre transgene selectively expressed in the epiblast from implantation stages onward (Hayashi et al. 2002). As expected, crossing the Sox2Cre transgene into the ROSA26 ${ }^{\mathrm{R}}$ conditional reporter background (Soriano 1999 ) results in rapid activation of LacZ expression throughout the entire epiblast from the earliest postimplantation stages onward (Fig. 2A-E; data not shown). The Sox2Cre transgene was first introduced into the Smad2 ${ }^{\text {Robm1 }}$ null strain (Waldrip et al. 1998), and next Sox2Cre/+;Smad2 $2^{\mathrm{Robm} 1 /+}$ and Smad2 $\mathrm{CA} / \mathrm{CA}$ animals were intercrossed.

Mutant Sox2Cre/+;Smad2 Robm1/CA embryos (designated conditional mutant embryos) gastrulate normally as assessed by expression of Fgf8 (Fig. 2F,G) and T mRNA (Fig. $2 \mathrm{H}, \mathrm{I})$ and at $7.5 \mathrm{~d}$ postcoitum (dpc) are morphologically indistinguishable from control littermates. However, by headfold stages $(8.5 \mathrm{dpc})$ the mutant embryos appear overtly abnormal, and specifically display a reduction in rostral neurectoderm tissue. To examine the onset and nature of these patterning defects, we analyzed the expression of diagnostic markers. Otx2 is widely expressed in the developing neurectoderm from pregastrulation stages onward and becomes confined to the forebrain and midbrain at early somite stages. Wild-type and mutant embryos display indistinguishable Otx2 expression patterns at $7.5 \mathrm{dpc}$ (Fig. 2J,K). However, $12 \mathrm{~h}$ later, the Otx2 expression domain is reduced in mutant embryos, and by $8.5 \mathrm{dpc}$ is confined to a small region at the most rostral extent of the CNS (Fig. 2L,M). Similar conclusions were reached analyzing Six3 (data not shown), a transcription factor expressed in the anterior neurectoderm.

To further evaluate anterior CNS defects, we analyzed Fgf8 expression, which is normally confined to the anterior neural ridge and defines the most rostral neurepithelium, as well as the isthmus marking the junction be- tween the developing midbrain and hindbrain. In conditional mutant embryos, Fgf8 expression is confined to a small patch of tissue representing the remnants of the isthmus (Fig. 2N,O), but fails to be expressed rostrally. Similarly, the ventral Shh domain is absent (Fig. 2R,T). In contrast, hindbrain formation is unaffected, as judged by expression of Krox20 (Fig. 2P,Q). Thus, we conclude that conditional loss of Smad2 in the epiblast has little effect on induction and initial specification of the neural plate. Rather, subsequent growth and anterior patterning are disturbed so that by $8.5 \mathrm{dpc}$ mutant embryos lack all prospective forebrain and most midbrain tissue.

In the mouse, initial anterior identity in the underlying epiblast is imposed by the AVE (Kimura et al. 2000; Perea-Gomez et al. 2002) and then, as gastrulation proceeds, it is stabilized by anterior streak derivatives. To evaluate the onset of anterior patterning defects in the Smad2 conditional mutant embryos, we first examined early postimplantation stages. As assessed by the expression of Cerl at $6.5 \mathrm{dpc}$ (Fig. 3A,B), the AVE is induced correctly and rotates appropriately in conditional mutants. GSC and Foxa2 expression normally marks both the AVE and the anterior PS (Fig. 3C,E). Interestingly, Gsc and Foxa2 expression in the AVE is unaffected, but mutant embryos only weakly express these markers in the posterior epiblast (Fig. 3D,F). This result suggests that the AVE functions normally and that loss of Smad2 in the epiblast selectively disrupts the patterning of the anterior PS. Therefore, the anterior defects observed in Smad2 conditional mutant embryos most likely result from impaired production of, or disrupted signaling within, the anterior streak derivatives such as the ADE, PCP, and node that guide anterior development after gastrulation.

Next, we assessed the development of the anterior streak derivatives. Blimp1, a zinc finger transcription factor belonging to the Krüppel family, is normally expressed in the AVE and ADE (Fig. 3G; de Souza et al. 1999). Blimp1 transcripts are present at barely detectable levels in the ADE of mutant embryos (Fig. 3H). Similarly, expression of the homeodomain gene Hex (Fig. 3K,L), a marker of the midline DE (Thomas et al. 1998), is markedly reduced. The DAN family member Cerl, which transiently marks the majority of nascent DE (Fig. 3I; Belo et al. 1997), is weakly expressed in fewer cells compared with wild type, and the Cerl ADE expression domain is virtually eliminated (Fig. 3J).

Figure 3. Mispatterning of the anterior streak derivatives in Sox2Cre/+;Smad2 $2^{\mathrm{CA} / \mathrm{Robm} 1}$ mutant embryos. Whole-mount in situ hybridization analysis of wild-type (WT) and Sox2Cre/+;Smad2 ${ }^{\mathrm{CA} / \text { null }}$ mutant embryos at $6.5 \mathrm{dpc}(A-F), 7.5 \mathrm{dpc}(G-R)$, and $7.75 \mathrm{dpc}$ $(S-X) .(A-D)$ As shown by Cerl and Foxa2 expression, the AVE is induced and rotates toward the presumptive anterior side of the embryo. $(C-F)$ However, the mutants express reduced levels of Foxa2 and Gsc in the anterior streak (indicated by red lines). Blimp1 normally expressed in the anterior mesendoderm $(K)$ is absent in the mutant $(L)$. Frontal views and transverse sections show that the Cerl expression domain in the definitive endoderm (DE) and mesoderm $(I$, sections I1 and I2) is reduced $(J$, sections $J 1, J 2)$. Hex $(K)$ and Foxa2 ( $M$, section $M 1)$, normally expressed in the midline DE, are absent in the mutants $(L, N$, section N1). Note that Foxa2 is expressed in the node in both wild-type (WT; $M$, section M2) and mutant ( $N$ and section N2) embryos. As shown by $S h h(O, P$, sections $\mathrm{O} 1$ and $\mathrm{P} 1$, respectively) and $\operatorname{Nodal}(Q, R)$ expression domains, the node is formed normally. However, $\operatorname{Shh}(O$, section $\mathrm{O} 2)$ and $G s c(S)$ expression domains marking the prechordal plate $(\mathrm{PCP})$ are missing in the mutant $(P$, cross-section $\mathrm{P} 2 ; \mathrm{T})$. Chd and Nog normally expressed in the midline and PCP $(U, W)$ are truncated anteriorly $(V, X)$. A, anterior; $\mathrm{P}$, posterior. Lateral views are shown with anterior to the left, with the exception of $I, I$, and $S-X$, which show frontal views. 
Foxa2 expression delineates the forming organizer and midline mesendoderm tissues in the 7.5-dpc embryo
(Fig. 3M). At 6.5 dpc, mutant embryos express Foxa2 only weakly in the anterior streak, but Smad2 loss has

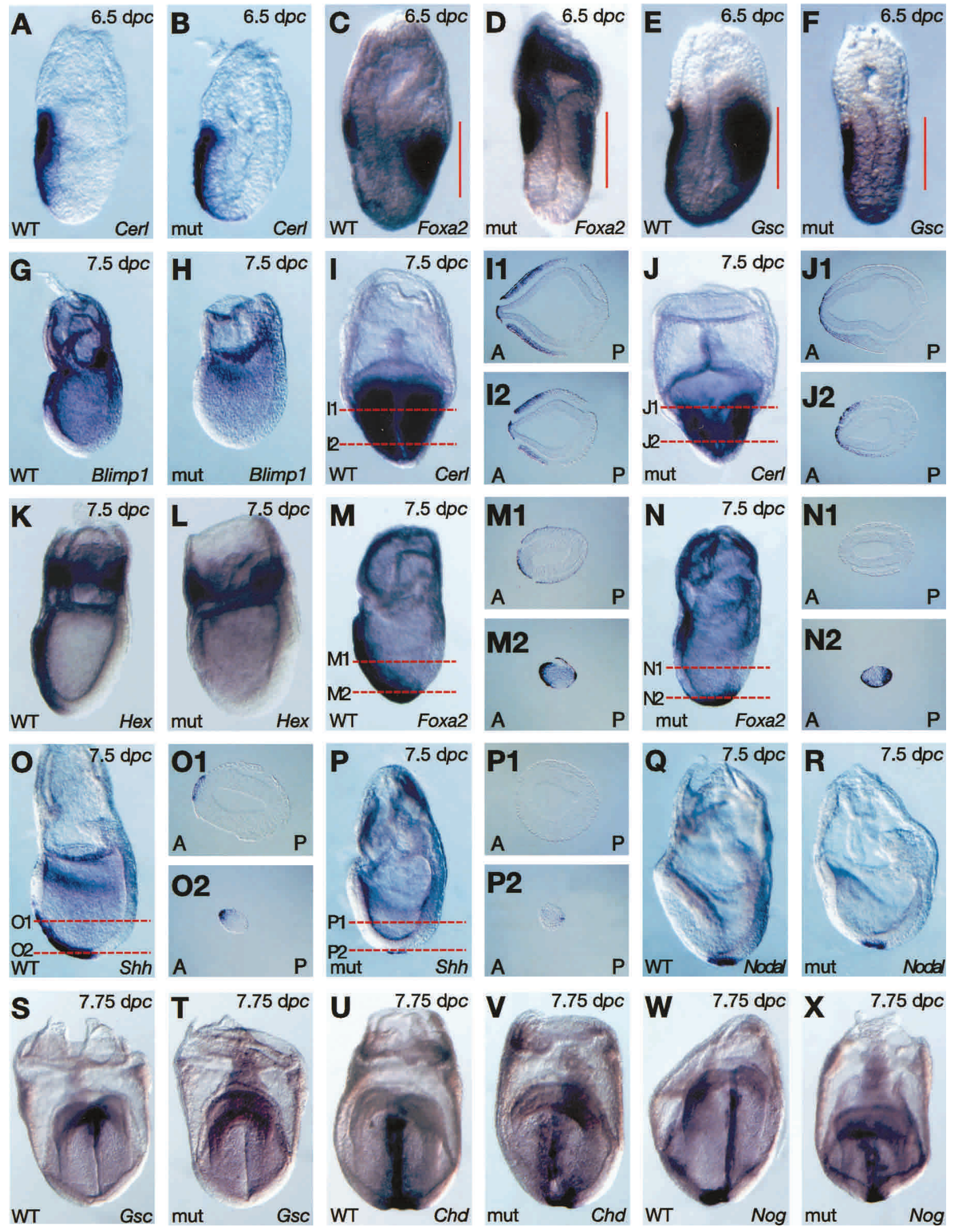

(Figure 3 legend on facing page) 
no noticeable effect on Foxa2 expression levels in the developing node. Strikingly, Foxa2 transcripts are not detected anterior to the node. Thus, it appears that anterior midline tissue is not specified. Alternatively, Foxa2 induction could potentially depend on Smad2 activity.

To further distinguish these possibilities, we analyzed Shh expression in the developing node and PCP at 7.5 dpc (Fig. 3O). As for Foxa2, in mutant embryos Shh is correctly expressed in the node but absent from the anterior midline tissue (Fig. 3P). To confirm that formation of the node and notochord is unperturbed in the conditional mutants, we examined Nodal (Fig. 3Q,R) and T (data not shown) expression, as well as Shh expression at $8.5 \mathrm{dpc}$ as a marker for the floor plate and notochord (Fig. 2R-T). These structures develop normally but the conditional mutant embryos display decreased levels of Shh transcripts (Fig. 2T,U).

The most rostral population of midline axial mesoderm gives rise to a histologically distinct population of cells termed the PCP (Sulik et al. 1994), which plays an important role as an organizing center responsible for patterning the developing brain. The loss of both Foxa2 and $S h h$ expression at $7.5 \mathrm{dpc}$ in the PCP progenitors emerging from the distal tip of the streak (Fig. 3) strongly suggests that Smad2 is essential for specification of this discrete cell type. Additional markers Gsc, and the BMP antagonists Chordin and Noggin diagnostic for the PCP, were examined in 7.75-dpc mutant embryos. As shown in Figure 3S-X, the prominent domain of GSC is lost, and expression of both Noggin and Chordin is severely attenuated along the midline in conditional mutant embryos.

To assess the functional consequences of perturbed expression of these midline markers, we examined Nodal expression in early somite stage embryos. Asymmetric expression of Nodal in the left lateral plate mesoderm (LPM) is known to be dependent on the presence of a functional "midline barrier". Consistent with disturbed function of the midline, a high proportion $(60 \% ; 6 / 10)$ of mutant embryos show bilateral Nodal expression (Fig. $2 \mathrm{~V}, \mathrm{~W})$. Thus, conditional loss of Smad2 selectively disrupts formation of the most anterior midline populations, namely, the PCP and ADE.

\section{Targeted removal of the PEE decreases Nodal expression in the primitive streak}

Smad2 and Smad3 are activated by type I receptors that bind TGF $\beta$ /activin/Nodal ligands (for reviews, see Massagué et al. 2000; Whitman 2001). To date, the only ligands of this class expressed in the early mouse embryo are Nodal (Zhou et al. 1993; Conlon et al. 1994) and Gdf1 (Wall et al. 2000). Gdf1 is required for establishment of the L-R axis (Rankin et al. 2000), whereas Nodal acts much earlier during A-P axis establishment (Conlon et al. 1994; Brennan et al. 2001). To test whether Nodal/ Smad2 signals are solely responsible for patterning of the anterior streak, we used a genetic strategy to selectively reduce Nodal transcription on the posterior side of the epiblast. The PEE element drives Nodal expression in the proximal epiblast cells prior to gastrulation and maintains Nodal expression along the PS at later stages (Norris and Robertson 1999). The 2.0-kb 5' region of the Nodal locus corresponding to the minimal PEE region, as defined in transgenic experiments (D.P. Norris and E.J. Robertson, unpubl.), was deleted in the germ line using a Cre-loxP approach to generate a novel allele, designated Nodal $^{\triangle \mathrm{PEE}}$ (Fig. 4). Whole-mount in situ hybridization experiments were used to examine Nodal expression levels at $6.0 \mathrm{dpc}$. At this stage, Nodal expression is normally confined to the VE and proximal epiblast. As shown in Figure 4E-F, Nodal levels are greatly reduced but not eliminated in embryos transheterozygous for the $N_{\text {Nodal }}{ }^{\mathrm{PEE}}$ and the Nodal ${ }^{413 . \mathrm{d}}$ null alleles (Conlon et al. 1994). Homozygous Nodal ${ }^{\mathrm{PEE} / \triangle \mathrm{PEE}}$ embryos express intermediate levels of Nodal mRNA (data not shown). At slightly later midstreak stages, low levels of Nodal mRNA are detected on the posterior side of the epiblast in Nodal $^{\triangle \mathrm{PEE} / 413 . \mathrm{d}}$ embryos compared with wild type (data not shown). At $7.5 \mathrm{dpc}$ in all classes of embryo, Nodal is expressed at wild-type levels in the developing node (data not shown).

\section{Defective formation of anterior streak derivatives} in Nodal NPEE/413.d $_{\text {embryos }}$

The functional impact of reduced Nodal signals in the PS was further assessed in test breeding experiments (data not shown). Nodal ${ }^{\mathrm{PEE} /+}$ animals are born at Mendelian frequencies, whereas only $50 \%$ of Nodal $^{\Delta \mathrm{PEE}}$ homozygotes survive to term. The remaining homozygous embryos die late in gestation or at birth as a result of a spectrum of complex defects, including craniofacial and heart abnormalities. There were no live born Nodal ${ }^{\Delta \mathrm{PEE} / 413 . d}$ transheterozygotes.

To determine the onset of developmental failure, we recovered embryos from early postimplantation stages onward. As shown in Figure 5, developmental defects in Nodal ${ }^{\mathrm{PEE} / 413 . \mathrm{d}}$ embryos recovered between 6.5 and 8.75 $\mathrm{dpc}$ are indistinguishable from those described earlier for Smad2 conditional mutants. Without exception, at 8.5 dpc, Nodal ${ }^{\triangle \mathrm{PEE} / 413 . \mathrm{d}}$ embryos develop severe anterior truncations. As judged by expression of Cerl (Fig. 5A,B) and Hex (Fig. 5C,D), the AVE is correctly specified. Decreased Nodal signals result in attenuation of Foxa2 and Gsc expression in the posterior epiblast (Fig. 5G-J). Formation of ADE and PCP progenitors is also defective. Thus, we observe altered Hex (Fig. 5M,N), Foxa2 (Fig. 5O,P), Cerl (Fig. 5Q,R), and Gsc (Fig. 5S,T) expression domains. Failure to form the PCP and ADE results in the inability to maintain anterior neural plate structures. Consequently, the forebrain and midbrain regions are not specified, as demonstrated by loss of Shh ventrally (Fig. 5U,V) and Fgf8 (Fig. 5W,X). In contrast, formation of the notochordal plate and its derivative, the notochord, are unaffected by reduced Nodal signals in the posterior epiblast. 


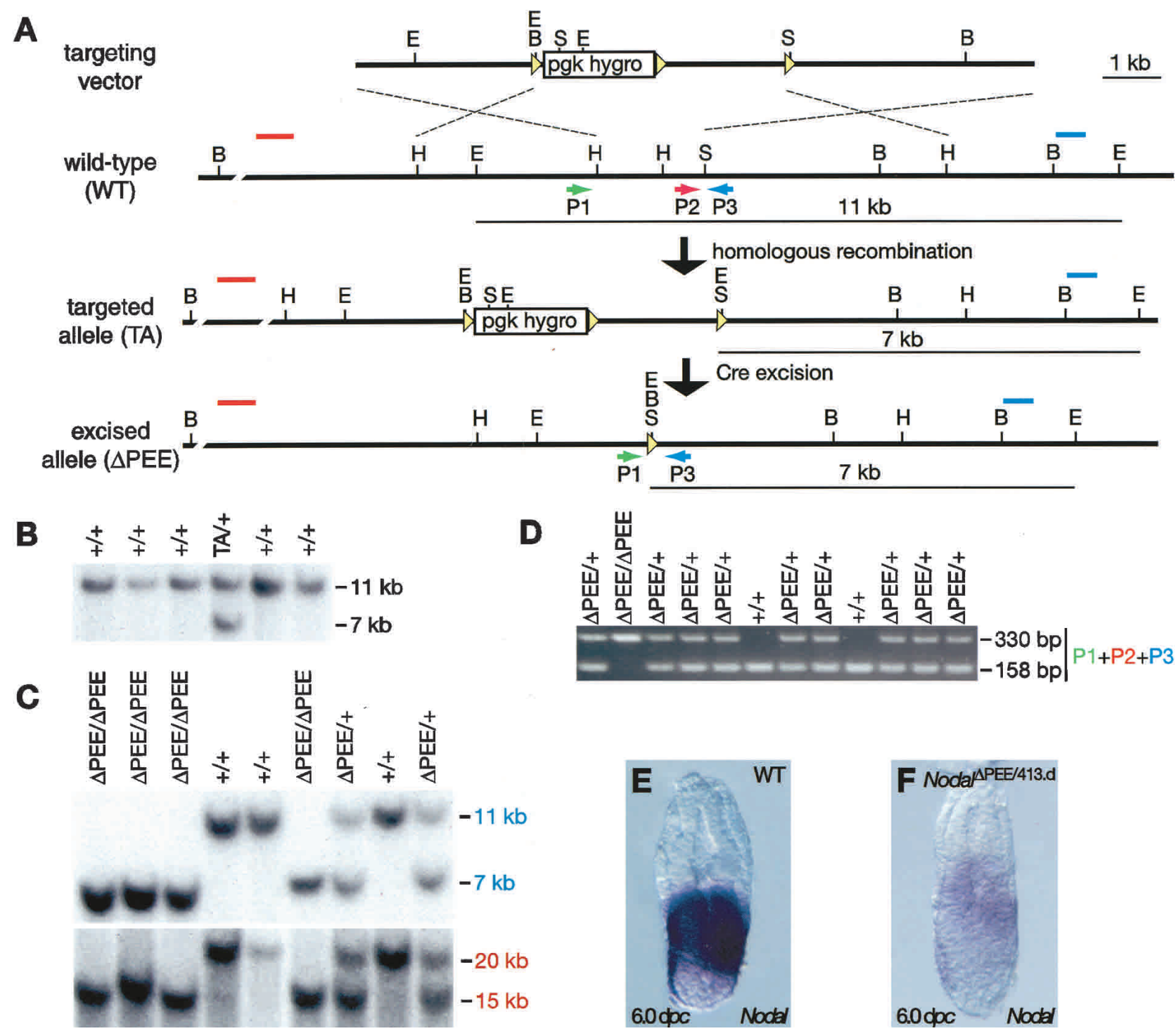

Figure 4. Generation of the Nodal ${ }^{\triangle \mathrm{PEE}}$ allele. $(A)$ Targeted deletion of the PEE element. Targeted clones identified using a $3^{\prime}$ external probe (blue line) were confirmed with a $5^{\prime}$ external probe (red line). The sizes of expected fragments are indicated. The arrows represent the primers used for genotyping. B, BamHI; E, EcoRI; H, HindIII; S, SpeI. (B) Southern blots of EcoRI-digested DNA from individual drug-resistant ES cell clones. The 3' external probe detects 11-kb wild-type (WT) and 9-kb targeted alleles. (C) Southern blots of EcoRIor BamHI-digested tail DNAs from intercross progeny using 3' (blue) or 5' (red) external probes, as indicated. $(D)$ PCR genotyping screen of an intercross litter yields predicted 330-bp (mutant) and 158-bp (wild-type, WT) products. (E,F,G) Whole-mount in situ hybridization of wild-type (WT; E) and transheterozygous Nodal ${ }^{\Delta \mathrm{PEE} / 413 . \mathrm{d}}(F)$ 6.5-dpc embryos showing decreasing Nodal expression levels in the epiblast and VE.

Defects in anterior streak specification compromise development of the anterior gut tube

As shown earlier, formation of the DE lineage is severely compromised in both categories of mutant embryo. Although the complete loss of this tissue is strongly suggested by the absence of Hex expression along the midline, Cerl, which normally delineates the DE, is diminished but not eliminated (Figs. 3, 5). To test whether these mutants fail to form DE or whether the tissue is simply mispatterned, we examined embryos histologically at $9.5 \mathrm{dpc}$. As shown in Figure 6, the gut tube forms but is very poorly elaborated in the anterior region. Nor- mally, the anterior gut tube extends to the level of Rathke's pouch (Fig. 6C). However, no gut tissue is evident in similar sections of mutant embryos (Fig. 6G,L). The anterior neurectoderm fails to proliferate and by $9.5 \mathrm{dpc}$ is filled with pyknotic nuclei (Fig. 6G,L). Gut tissue is absent anterior to the level of the heart. The heart tube is abnormally looped, and the pericardium was often enlarged and edematous (Fig. 6I,N), most likely reflecting failure of the endoderm to contribute to the normal induction and patterning of the heart field. In contrast, a distinct hindgut is present, the notochord forms normally, and paired somites are present on either side of the midline (Fig. 6I,J,N,O). 

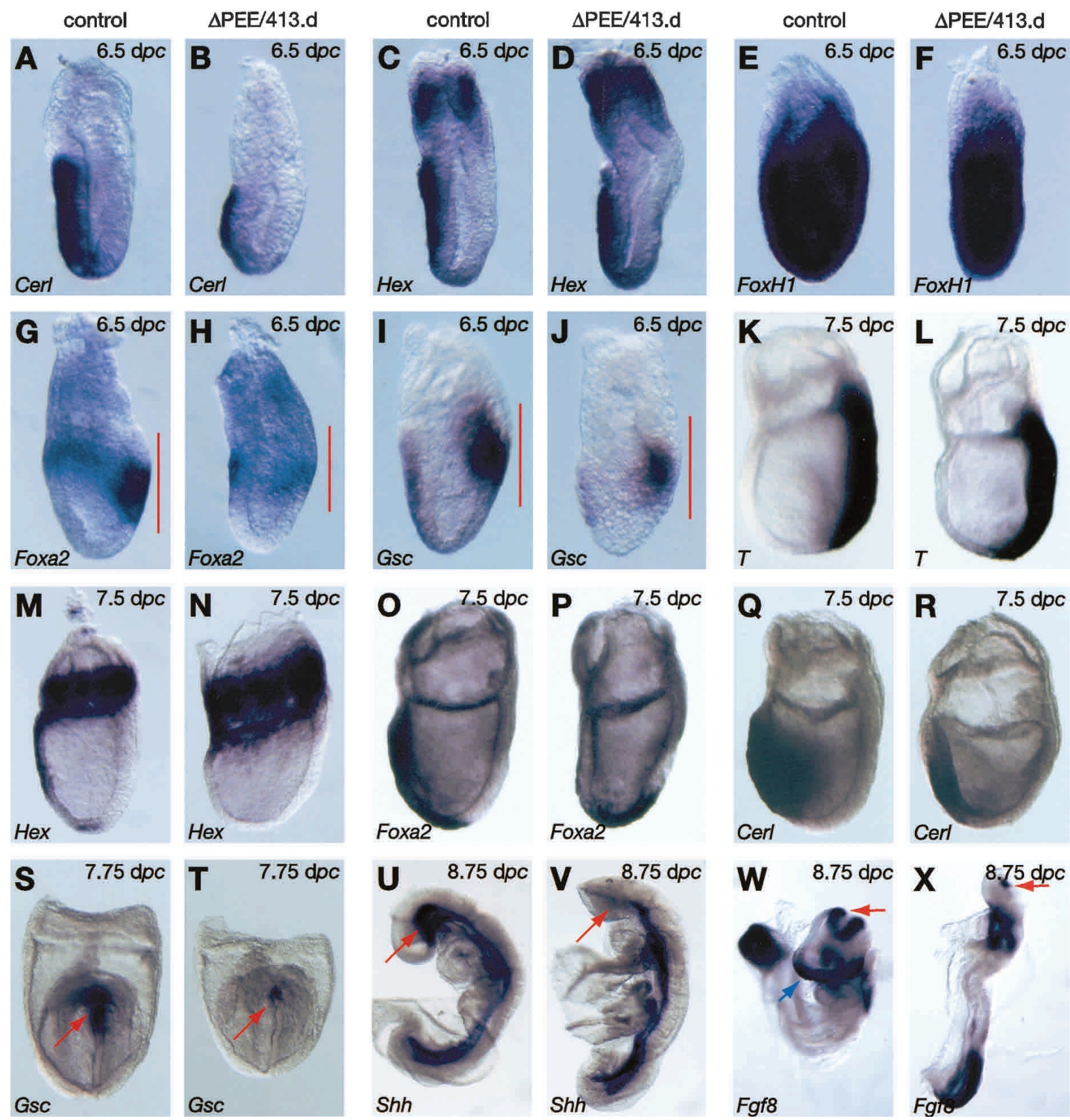

Figure 5. Nodal ${ }^{\Delta \mathrm{PEE} / 413 . \mathrm{d}}$ mutants closely resemble Sox2Cre/+;Smad2CA/Robm1 mutant embryos. Whole-mount in situ analysis hybridization of wild-type (WT) and Nodal ${ }^{\Delta \mathrm{PEE} / 413 . \mathrm{d}}$ mutant embryos at $6.5 \mathrm{dpc}(A-T), 7.5 \mathrm{dpc}(K-R), 7.75 \mathrm{dpc}(S, T), 8.5 \mathrm{dpc}(U-V)$, and 8.75 $\mathrm{dpc}(W-X)$. The AVE is specified and rotates toward the anterior side of the embryo $(A-D, G-I)$, as shown by Cerl $(A, B), \operatorname{Hex}(C, D), F o x a 2$ $(G, H)$, and $G s c(I, J)$ expression. Reduced Nodal activity has no effect on expression of FoxH1, a coeffector of Nodal signaling $(E, F)$, or streak induction and elongation, as shown by $T$ expression $(K, L)$. As for the Smad2 conditional mutant embryos, expression of Foxa2 $(G, H)$ and $G s c(I, J)$ is decreased in the anterior streak (indicated by red lines) compared with wild type (WT). Consequently, expression of the midline definitive endoderm markers $\operatorname{Hex}(M, N)$ and $\operatorname{Fox} a 2(O, P)$ is absent and the Cerl expression domain is reduced $(Q, R)$. As for Smad2 conditional mutants, the Nodal ${ }^{\mathrm{PEE} / 413 . \mathrm{d}}$ mutants express Foxa2 in the forming node. $(S, T)$ Frontal views show that GsC is absent or severely reduced in the prechordal plate in Nodal ${ }^{\Delta \mathrm{PEE} / 413 . \mathrm{d}}$ mutant embryos. $(U-W)$ Truncation of the anterior part of the brain is revealed at $8.5 \mathrm{dpc}$ by the absence of the ventral forebrain domain of $S h$ (red arrow in $U, V$ ) and loss of $F g f 8$ marking the anterior neural ridge (blue arrow in $W) .(W, X)$ However, a residual Fgf8 isthmic region (red arrow) is still present in the mutants. 

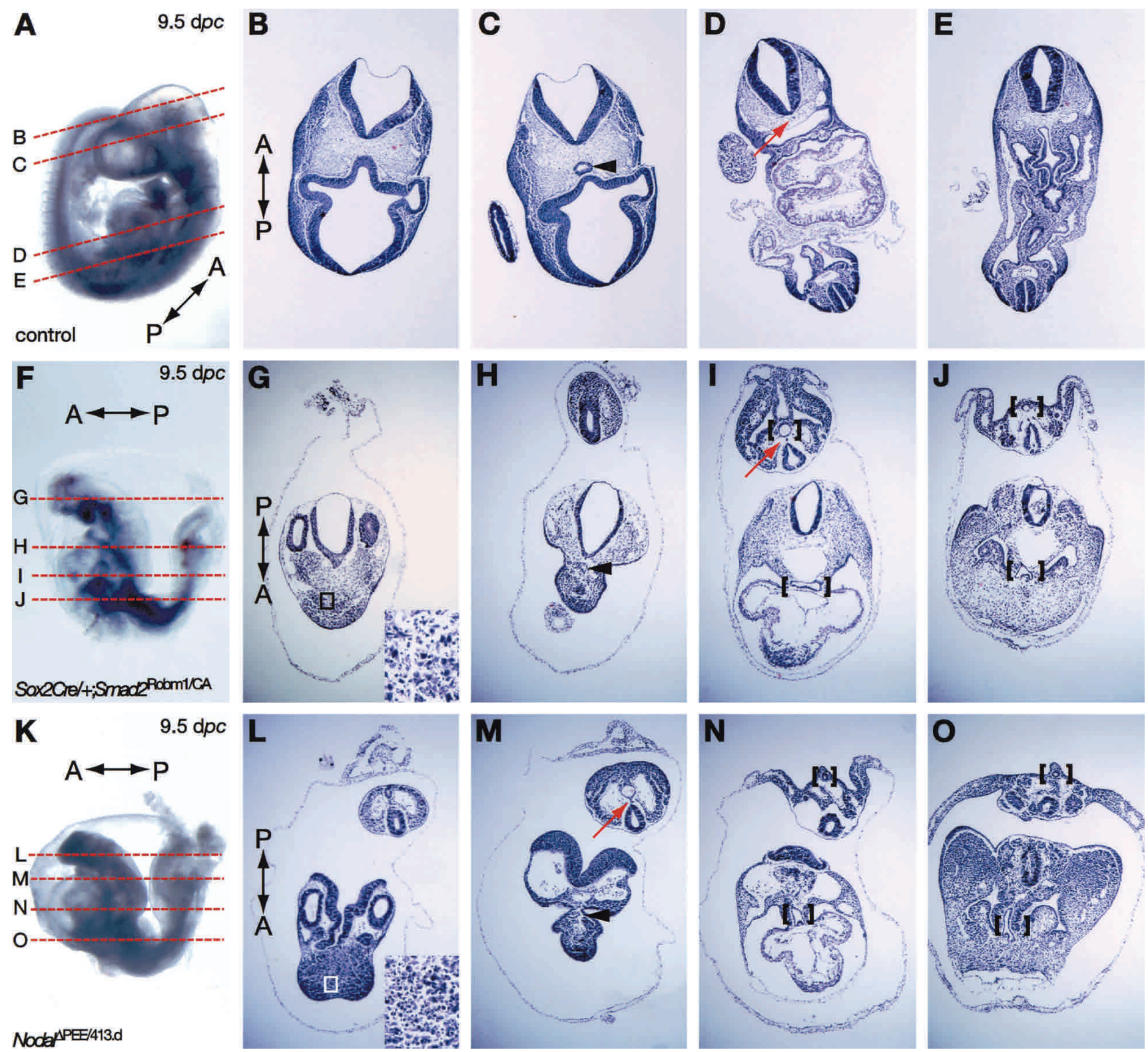

Figure 6. Gut tube formation in the Nodal ${ }^{\Delta \mathrm{PEE} / 413 . \mathrm{d}}$ and Sox2Cre/+;Smad2 ${ }^{\mathrm{CA} / \mathrm{Robm} 1}$ mutant embryos. Wild-type (WT; A-E), Sox2Cre/ +;Smad2 ${ }^{\mathrm{CA} / \mathrm{Robm} 1}$ mutant $(F-J)$, and Nodal ${ }^{\Delta \mathrm{PEE} / 413 . \mathrm{d}}$ mutant $(K-O)$ 9.5-dpc embryos. $(B, G, L)$ Sections at the level of the head show loss of anterior neurectoderm in both categories of mutant embryos and, as shown in high magnification in lower left $(G, L)$, residual anterior tissue contains many pyknotic cells. $(C)$ Normally the gut tube (arrowhead) extends into the head territories). In both classes of mutants $(H, M)$ the anterior gut tube (arrowhead) is shortened and fails to extend anterior to the heart $(D, I, N)$. Sections at the level of the heart reveal that the neural tube is highly abnormal. A poorly elaborated gut tube-like structure is present (brackets) but the heart fails to undergo correct looping morphogenesis and has an abnormal trifolium shape. $(E, J, O)$ Sections at the level of the posterior somites. (E) In the wild-type (WT) embryo, the somites have already started to differentiate into dermomyotome and sclerotome. Distinct bilateral somites and hindgut (brackets) are present in both categories of mutant embryos. $(I, M)$ The notochord (red arrow) is clearly detectable in the Smad2 and Nodal ${ }^{\Delta \mathrm{PEE} / 413 . \mathrm{d}}$ mutant embryos.

\section{Extraembryonic endoderm contributes to gut formation in Smad2 conditional mutant embryos}

The DE emerging from the anterior streak during gastrulation normally displaces the VE into the visceral yolk sac (Thomas and Beddington 1996). In the absence of Nodal/Smad2 signals, the gut tube evident at $9.5 \mathrm{dpc}$ in mutant embryos might be derived in part from the ex- traembryonic VE as a consequence of improper definitive endoderm specification. Consistent with this idea, using $H n f 4 \alpha$ as a marker of extraembryonic endoderm, we consistently observed that the $H n f 4 \alpha$ positive cell population extended more distally in Smad2 conditional mutants in comparison with wild-type embryos (Fig. 7A,D). Thus, it appears that VE derivatives become incorporated into embryonic gut tissues. 
To unequivocally determine the origins of the gut endoderm, we used an in vivo fate mapping strategy. The ROSA26 ${ }^{\mathrm{R}}$ conditional allele (Soriano 1999) was intro- duced into the Smad2 ${ }^{\mathrm{CA}}$ background. Smad2 ${ }^{\mathrm{CA} / \mathrm{CA}}$; ROSA $26^{\mathrm{R} / \mathrm{R}}$ and Sox $2 \mathrm{Cre} /+$ Smad2 $2^{\mathrm{Robm} 1 / \mathrm{CA}}$ mice were then intercrossed. Using this strategy, the Sox2Cre
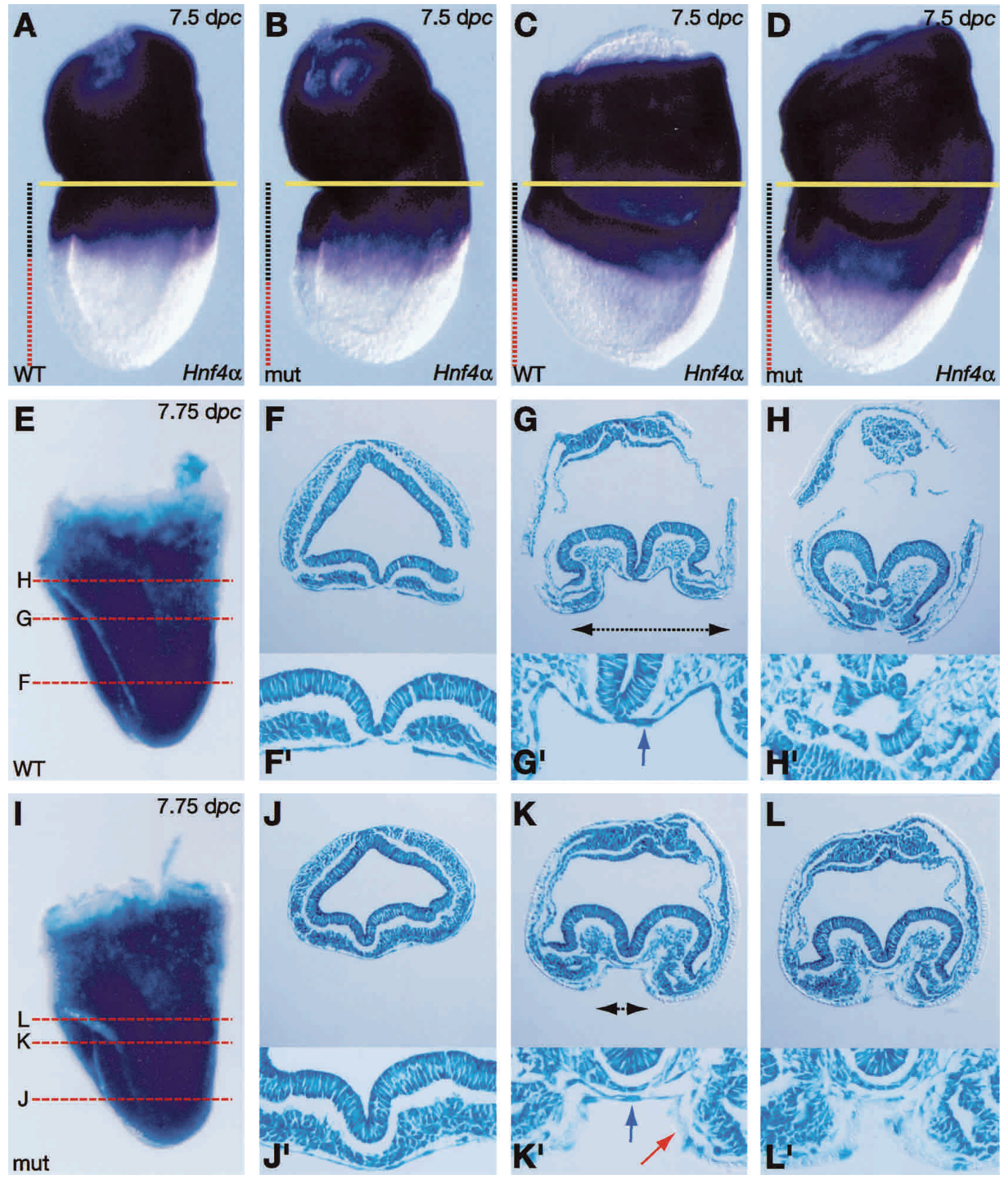

Figure 7. VE-derived cells contribute to the gut tube in Sox2Cre/+;Smad2 ${ }^{\mathrm{CA} / \text { Robm1 }}{ }^{1}$ mutants. $(A-D)$ Whole-mount in situ hybridization analysis of $H n f 4 \alpha$ expression. In Smad2 conditional mutant embryos, the VE domain is incompletely displaced toward the proximal extraembryonic region. The boundary separating the extraembryonic and embryonic regions is indicated by a horizontal yellow line. The extent of $H n f 4 \alpha$ expression relative to the distal nonstaining domain is indicated by black and red vertical lines, respectively. (E-L) Sections of X-gal stained Sox 2 Cre $/+; R O S A 26^{\mathrm{R} /+}$ and Sox 2 Cre $/+;$ Smad2 ${ }^{\mathrm{CA} / \mathrm{Robm} 1}{ }_{;}$ROSA26 ${ }^{\mathrm{R} /+}(I-L)$ 7.75-dpc embryos. The lateral limit of the definitive endoderm layer lining the foregut pocket (black arrows) is severely diminished in mutant $(K)$ compared with wild-type $(G)$ embryos. Wild-type definitive endoderm is exclusively composed of flattened LacZ-marked epiblast-derived cells $(F-H ;$ magnification in $F^{\prime}-H^{\prime}$; blue arrow in $G^{\prime}$ ), whereas the majority of cells in the mutant, especially in the most anterior part of the embryo, fail to express $L a c Z(K, L) .\left(K^{\prime}, L^{\prime}\right)$ Higher-magnification views reveal the presence of intruding cuboidal endoderm (red arrow), morphologically similar to extraembryonic VE that predominates over midline epiblast-derived LacZ-marked cells (blue arrow in $K$ ). 
transgene activates the $R O S A 26^{\mathrm{R}}$ reporter allele exclusively in derivatives of the epiblast (Fig. 2A-E) and not in the VE and extraembryonic ectoderm. As expected, in control embryos, the gut tube is uniformly composed of LacZ-positive DE cells (Fig. 7E-H). In contrast, the anterior gut tube in conditional mutants predominantly comprises LacZ-negative VE derivatives (Fig. 7K,L). Moreover, the mid- and posterior gut tissues are a mosaic of both VE-derived and epiblast-derived cell populations (Fig. 7J). Thus, Smad2-deficient epiblast cells have the ability to form definitive endoderm-like cells, but fewer progenitors are specified. These reduced numbers of ADE cells fail to efficiently displace primitive VE, so that VE cells become incorporated into the superficial tissue layer overlying the embryonic region. These persisting VE-derived cells lack the ability to maintain expression of key regulators including Hex and Cerl, and are functionally unable to substitute for the DE in patterning the neurectoderm, resulting in complex tissue defects that selectively disrupt formation of anterior structures.

Thus Smad2-deficient epiblast cells have the ability to form DE-like cells, but fewer progenitors are specified. Consequently, the VE is not efficiently displaced. These persistent VE cells fail to express a number of key regulators including Hex and Cerl and are unable to functionally substitute for the DE in patterning the neurectoderm.

Smad2 conditional mutant embryos lacking one copy of Smad3 show loss of node-derived structures

The forkhead transcription factor FoxH1 complexes with Smad2 and Smad4 to mediate Nodal signaling (for review, see Whitman 2001). Unlike mutant embryos described here, FoxH1-/- embryos lack both axial mesendoderm as well as the node and its derivatives (Hoodless et al. 2001; Yamamoto et al. 2001). One possibility is that Smad3, a closely related effector molecule broadly coexpressed with Smad2 (Tremblay et al. 2000), can also function downstream of Nodal signals. To test this possibility, we introduced a Smad3 ${ }^{\text {null }}$ allele (Datto et al. 1999) into the Sox2Cre/+;Smad2 ${ }^{\text {Robm1/CA }}$ background. At $8.5 \mathrm{dpc}$, Sox2Cre/+;Smad2 Robm1/CA $;$ Smad3 ${ }^{\text {null/+ }}$ embryos develop severe anterior truncations (Fig. 8E-G) and heart malformations. The anterior gut is absent (Fig. 8B,F) and, interestingly, the somites are fused across the midline (Fig. 8G), a feature associated with loss of the node and notochord. To further evaluate development of axial structures, we next assessed Foxa2 and Shh expression. Foxa2 expression in the node and its derivatives (Fig. 8D) is completely lost in Sox2Cre/+; Smad2 ${ }^{\text {Robm1/CA }} ;$ Smad3 ${ }^{\text {null/+ }}$ embryos (Fig. $8 \mathrm{H}$ ). Only a weak residual signal is detectable at the level of the foregut-like and hindgut-like structures (data not shown). Similarly, Shh is only weakly expressed at the level of the hindgut-like pocket (data not shown). Thus Sox2Cre/ +;Smad2 ${ }^{\text {Robm1/CA }}$;Smad3 ${ }^{+/ \text {null }}$ embryos closely resemble FoxH1-l- mutants. The more severe phenotype observed in Smad2 conditional mutants lacking one copy of
Smad3 suggests that graded Nodal/Smad2 signals govern specification of anterior streak derivatives during gastrulation.

\section{Discussion}

Here we demonstrate that targeted deletion of Smad2 function in the early epiblast or decreased Nodal signals in the posterior epiblast and PS both cause remarkably similar patterning defects that are restricted to anterior tissues derived from the mouse "organizer". In both classes of mutants, the PS forms and elongates normally. Mesoderm formation along the streak is also unaffected. Moreover, the AVE rotates to overlie the anterior epiblast and functions correctly. However, the distal streak population that encompasses the gastrula organizer displays reduced expression of Gsc and Foxa2. At late streak stages, expression of midline markers (Foxa2, Hex) is abolished, and Cerl expression normally marking a broader DE domain is also diminished. The neural plate is specified correctly. However, abnormal development and function of the anterior mesendoderm causes the most anterior structures of the brain to lose their identity and regress by apoptosis. Loss of Smad2 signaling in the anterior streak also results in a severe depletion of DE progenitors that compromises the ability of the DE to displace VE cells and results in a gut tube comprising both DE and VE derivatives. In striking contrast, formation of the node and its derivatives is unaffected. These findings conclusively demonstrate that Nodal/Smad2 signals govern allocation of the axial mesendoderm precursors that selectively give rise to the ADE and PCP mesoderm.

Previous studies demonstrate that Nodal signals relayed from the epiblast activate Smad2 in the VE in order to establish the AVE, initiating the specification of anterior character (Brennan et al. 2001). In the absence of Smad2 in the VE, anterior development never initiates and posteriorizing signals predominate, causing the epiblast to become diverted exclusively into extraembryonic mesoderm (Waldrip et al. 1998; Heyer et al. 1999). Here we extend these previous analyses and show that Smad2 activity in the epiblast is not required for formation and elongation of the PS and that extraembryonic, paraxial, and axial mesoderm, including the node and notochord, develop normally. However, aberrant Noggin and Chordin expression patterns provide compelling evidence that the midline is not fully functional. This finding is further supported by the observation that Nodal, normally confined to the left LPM by the midline barrier, is expressed bilaterally in a high proportion of conditional mutant embryos. In contrast, strongly chimeric embryos predominantly composed of Smad2-deficient cells display a histologically normal midline, but the DE is almost entirely derived from wild-type host cells. In this case, the midline barrier functions normally and Nodal activation is confined to the left LPM (Tremblay et al. 2000). Taken together, these observations lead us to conclude that Smad2-dependent signals from the DE and 
Vincent et al.
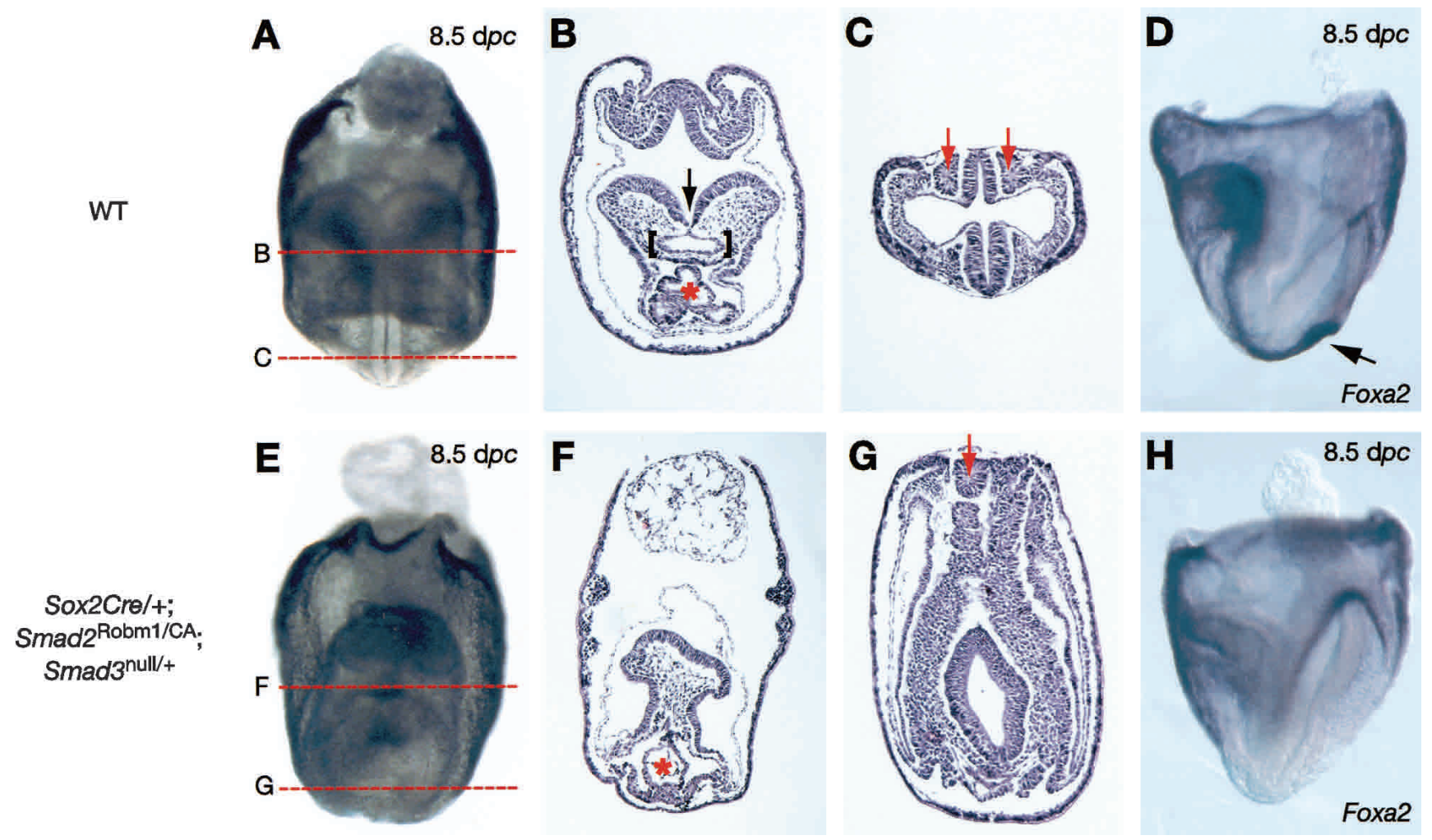

Figure 8. Decreasing Smad3 expression in Smad2 conditional mutant embryos results in loss of axial structures. $(A)$ Frontal view of a wild-type (WT) 8.5-dpc embryo showing the presence of a midline. $(B, C)$ Hematoxylin-eosin (HE)-stained sections of $A$. The level of the sections is indicated in $A$. (E) Frontal view of a Sox $2 C r e /+; S m a d 2^{\text {Robm1/CA }} ;$ Smad $3^{\text {null/++ }} 8.5$-dpc mutant embryo showing absence of midline structures. $(F-G)$ HE-stained sections of $E$. The level of sections is indicated in $E$. $(D, H)$ Foxa2 whole-mount in situ analysis of wild-type $(\mathrm{WT} ; D)$ and Sox2Cre/+;Smad2 ${ }^{\mathrm{Robm} 1 / \mathrm{CA}} ;{ }_{;} \operatorname{Smad} 3^{\text {null/++}}(H) 8.5 \mathrm{dpc}$ embryos. $(F)$ In the Sox2Cre/+;Smad2 ${ }^{\mathrm{Robm} 1 / \mathrm{CA}} ;$ Smad3 $^{\text {null/+ }}$ mutant embryo, the neural tissues remain as a neural plate, the heart (red asterisk) has not looped, and the foregut pocket (brackets in $B$ ) is absent. The absence of a midline is shown by the unfolded neural tube (arrow; cf. $F$ and $B$ ) and the fused somites (red arrows; cf. $G$ and $C$ ). Foxa2 is expressed in the node (arrow in $D$ ) and in the axial mesendoderm of the wild-type (WT) embryo. Neither of these expression domains is present in Sox $2 \mathrm{Cre} /+; \operatorname{Smad} 2^{\mathrm{Robml} / \mathrm{CA}} ; \operatorname{Smad} 3^{\text {null/++}}$ mutant embryos, confirming the absence of a node and its derivatives.

PCP are required for normal function of midline structures.

It is well known from studies in Xenopus that vertebrate head development occurs as a two-step induction process (for review, see Stern 2001). In mouse, induction of the AVE shortly after implantation initiates a Smad2dependent pathway that inhibits the posteriorizing influence of Nodal signals on the anterior side of the epiblast and maintains a labile, preneural and preforebrain state (Kimura et al. 2000; Brennan et al. 2001; PereaGomez et al. 2002). Following the onset of gastrulation, a second signal from the anterior streak-derived mesendoderm is required to maintain and stabilize the neural and forebrain region. Here we show in embryos selectively lacking Smad2 function in the epiblast that the AVE is induced and the neural plate is specified as judged by $\mathrm{Otx} 2$ and Six 3 expression. However, formation of the $\mathrm{PCP}$ and the ADE precursors is severely compromised. Consequently, anterior specification is not maintained. The anterior neurectoderm is deprived of essential signals, and all forebrain markers are lost or reduced. Thus, the Nodal/Smad2 signaling cascade specifies the ante- rior mesendoderm required for maintaining forebrain specification.

The role of the anterior mesendoderm in maintaining the anterior neural plate/forebrain domains has previously been described in studies of Foxa2 and Hex mutant embryos (Dufort et al. 1998; Martinez Barbera et al. 2000). Here we demonstrate that the Nodal/Smad2 pathway acts upstream of these genes in determining cell fate. One possibility is that these genes are direct targets of Nodal/Smad2 signaling. Consistent with this, the AVE and ADE specific enhancer mapped within the Hex locus contains Foxa2 binding sites and sequences bound by SIP-1, a protein that interacts with Smad2 (Rodriguez et al. 2001). Similarly, a Smad2 responsive enhancer has been characterized within the Xenopus XFKH1 locus (Howell and Hill 1997). However, studies to date have failed to identify FoxH1 and/or Smad binding sites within the mouse Foxa2 locus (Sasaki and Hogan 1996; Hoodless et al. 2001), and Foxa2 is weakly induced in the anterior streak and PCP tissues of FoxH1 mutant embryos (Hoodless et al. 2001; Yamamoto et al. 2001). These findings suggest that in mouse, 
Foxa2 may be a secondary target of the Nodal/Smad2 pathway.

Interestingly, Foxa2 mutant embryos lack a node and midline derivatives (Ang and Rossant 1994; Weinstein et al. 1994). Similarly, chimeric embryos generated by aggregating tetraploid wild-type embryos with Foxa2-deficient ES cells (Dufort et al. 1998) not only lack a node and midline derivatives, but also lack foregut and midgut tissues and contain only a rudimentary hindgut. Furthermore, deletion of Foxa2 in the axial mesendoderm leads to loss of the most anterior structures of the brain (Hallonet et al. 2002). The present results demonstrate decreased Nodal signaling in the anterior streak downregulates Foxa2 expression. Considering that node morphogenesis is unperturbed, we conclude that residual Foxa2 expression in the anterior streak is sufficient to promote node formation. In contrast, Arkadia mutants fail to activate Foxa2 in the anterior streak, and these embryos lack both a node and axial mesendoderm (Episkopou et al. 2001). Similarly, loss of Foxa2 expression and node-derived structures was observed in Smad2 conditional mutant embryos lacking one copy of Smad3. Collectively, these observations implicate graded Foxa2 requirements during specification of the anterior streak derivatives. Thus, it appears that PCP and ADE progenitors selectively require increased Nodal/Smad2/Foxa2 expression. Similarly, pharynx organogenesis in Caenorhabditis elegans is controlled by pha-4, a Foxa2 homolog, and sequential activation of diverse downstream target genes by pha-4 is regulated by the relative affinities of PHA-4 for variants of its canonical binding site (Gaudet and Mango 2002). Here we demonstrate that reduced Foxa2 expression selectively disrupts allocation of cells into the PCP and ADE cell lineages. It is tempting to speculate that activation of distinct Foxa2 target genes guiding cell fate decisions within the mouse organizer is similarly regulated as a result of different affinities for activated Foxa2 complexes.

Nodal expression in the epiblast becomes restricted to the presumptive posterior side of the embryo shortly before the onset of gastrulation (Varlet et al. 1997). Two enhancers, a conserved intronic enhancer (ASE) containing binding sites for the FoxH1 transcription factor and the PEE element, contribute to Nodal expression in the epiblast (Norris and Robertson 1999). The ASE autoregulatory enhancer promotes activation and upregulation of Nodal expression in the epiblast and VE. Deletion of this element attenuates Nodal signals in the epiblast, and allows specification of the AVE but fails to promote cell movements required to convert the $\mathrm{P}-\mathrm{D}$ to the A-P axis (Norris et al. 2002). Here we show that decreased Nodal expression in the posterior epiblast of Nodal ${ }^{\Delta \mathrm{PEE} / 413 . \mathrm{d}}$ mutant embryos causes loss of PCP and a reduction in DE progenitors. Thus, we conclude that the strength of Nodal signaling selectively regulates cell fate decisions in the streak.

In lower vertebrates, distinct aspects of early embryonic patterning require graded doses of Nodal signaling. For example, in Xenopus, increased levels of Nodal signaling are required for formation of dorsal mesoderm, whereas lower levels are sufficient for development of ventral mesodermal cell types (Agius et al. 2000). Similarly, in Zebrafish, specification of the PCP requires high levels of Nodal, whereas notochord specification occurs at lower levels (Gritsman et al. 2000). In Xenopus embryos, Activin concentration gradients are perceived by the number of occupied receptors per cell, and activation of target genes like Xbra or Xgsc depends on the degree of Activin receptor occupancy (Dyson and Gurdon 1998). A similar mechanism may be responsible for interpreting Nodal signaling thresholds and activating subsets of target genes in discrete cell types during distinct aspects of Nodal-dependent A-P and L-R patterning. Moreover, sustained Nodal/Smad2 signaling may be required to maintain specific programs of gene expression over time. For example, in Nodal ${ }^{\Delta 600 / \Delta 600}$ mutant embryos in which asymmetric Nodal expression is greatly reduced, Lefty2 is not activated in the left LPM, whereas Pitx2 is robustly expressed, although with a slightly delayed onset (Norris et al. 2002). Because both Lefty2 and Pitx2 are direct Nodal targets, differential gene induction appears to reflect locus specific response to differing levels of activated Smad complexes. Interestingly, although activation of Pitx 2 transcription is known to be mediated via a FoxH1 responsive enhancer, a second regulatory element that specifically binds Nkx2.1 at slightly later developmental stages is required for continued maintenance of transcription (Shiratori et al. 2001).

Nodal signals play a conserved role in specification of the DE lineage in vertebrates (David and Rosa 2001; Lowe et al. 2001; Norris et al. 2002). In mutant embryos described here, we fail to observe expression of midline DE markers (Hex, Foxa2). Cerl, a broader DE marker, is only weakly expressed and is absent from the ADE. However, histological analysis clearly shows that ventral closure occurs and a gut structure is present. In chimeric embryos, neither Smad2 ${ }^{-/-}$nor FoxH1 $1^{-/-}$ES cells contribute to the gut tube, strongly suggesting that both activities are necessary for DE specification (Tremblay et al. 2000; Hoodless et al. 2001). Here we show that the anterior part of the gut tube is truncated and mainly derives from extraembryonic VE cells, whereas the midgut and hindgut contain mainly epiblast derivatives. These results demonstrate that the VE compensates physically but cannot functionally replace DE cells. Strikingly, despite numerous genes expressed in common in both extraembryonic and DE cell populations, the VE appears to lack signaling capabilities. It will be interesting to identify DE-specific signals and learn more about the mechanism by which they pattern the overlying neuroectoderm.

\section{Materials and methods}

Generating a Smad2 conditional allele

The Smad2 ${ }^{\mathrm{CA}}$ allele was generated by flanking the first coding exon with loxP sites (Fig. 1A). The targeting construct contains a 5-kb (SpeI-EcoRI) 5' and a 4.9-kb (EcoRI-XhoI) 3' homology arm containing a loxP site that replaces a PstI site with an EcoRI 
site, and loxP flanked pgk-neomycin and pgk-dta selection cassettes (Fig. 1A). Linearized vector was electroporated into CCE ES cells, and drug-resistant colonies were genotyped by Southern blot analysis as described (Waldrip et al. 1998). Approximately $6 \%$ of the clones were correctly targeted. The pgk-neomycin cassette was excised by transient expression of Cre recombinase. Two independent Smad2 ${ }^{\mathrm{CA} /+}$ ES cell clones were used to generate germ line chimeric mice that were subsequently bred to C57BL/6 mice.

\section{Generation of the Nodal ${ }^{\triangle P E E}$ allele}

The PEE element corresponds to a 2-kb (HindIII-SpeI) region located $\sim 10 \mathrm{~kb} \mathrm{5}$ ' of the first exon of Nodal (Norris and Robertson 1999). The PEE targeting vector was designed using a conditional strategy (Fig. 4A) and contains a 3-kb (HindIII-HindIII) $5^{\prime}$ and a 6.2-kb (HindIII-EcoRI) 3' homology arm. The SpeI site within the $3^{\prime}$ arm was replaced by a loxP site and an EcoRI site. The homology arms were subcloned into a vector containing a loxP-flanked pgk-hygro cassette and hsv-tk and pgk-dta negative selection cassettes. Linearized vector was electroporated into CCE cells and drug-resistant colonies were screened by Southern blot analysis using a $3^{\prime}$ external (BamHI-PstI) probe. Approximately $5 \%$ of the clones were correctly targeted. The pgk-hygro cassette and 2-kb PEE element were excised from targeted clones by transient expression of Cre recombinase. Resulting subclones were screened by PCR and Southern analysis using 5' (SacI-PstI) and 3' external probes. Four correctly excised ES cell clones were used to generate germ line chimeric mice that were subsequently bred to C57BL/6 mice.

\section{Genotyping procedures}

Mice were genotyped by PCR screening of genomic tail DNA. Embryos were individually genotyped prior to whole-mount in situ hybridization and/or histological analysis by digesting a fragment of extraembryonic tissue in $20-50 \mu \mathrm{L}$ of $1 \times$ Perkin Elmer PCR buffer (Roche) containing $0.45 \%$ NP-40, $0.45 \%$ Tween 20, and $1 \mu \mathrm{g} / \mu \mathrm{L}$ Proteinase $\mathrm{K}$ for $9 \mathrm{~h}$ at $56^{\circ} \mathrm{C}$. Lysates were boiled and $0.5-1 \mu \mathrm{L}$ was used for PCR analysis.

The Noda1 ${ }^{413 . \mathrm{d}}, S \mathrm{Smad2} 2^{\mathrm{Robm} 1}$, and ROSA26 $6^{\mathrm{R}}$ alleles and the Cre transgene were genotyped as described (Conlon et al. 1994; Vidal et al. 1998; Waldrip et al. 1998; Soriano 1999). Primers used to genotype the $\mathrm{Smad} 2^{\mathrm{CA}}$ and $S \mathrm{Smad} 2^{\mathrm{CD}}$ alleles were as follows (Fig. 1A): S1, 5'-CCCGGTAAATCTACCCTAG-3'; S2, 5'-GCTTAAAAGTCACTACTCAAG-3'; S3, 5' -TTTCAAAAC TATATTTGCCCAAG-3'.

The S2 + S3 pair amplifies 220-bp and 320-bp fragments for the wild-type and the $S$ mad2 ${ }^{\mathrm{CA}}$ alleles, respectively. The S1 + S3 pair amplifies 650-bp, 700-bp, and 200-bp fragments for the wild-type, Smad2 ${ }^{\mathrm{CA}}$, and $\operatorname{Smad} 2^{\mathrm{CD}}$ alleles, respectively.

The Nodal ${ }^{\triangle \mathrm{PEE}}$ allele was genotyped using the following primers (Fig. 4A): P1, 5'-AGTGCTGGGATCACAGAAG-3'; P2, 5'-GAGATAGGTCTTGTGTGGC-3'; P3, 5' -TATGATAATGA TCAGGTCAGG-3'.

The combination of the $\mathrm{P} 1, \mathrm{P} 2$, and $\mathrm{P} 3$ primers amplifies 160 bp and 330-bp fragments from the wild-type and the Nodal ${ }^{\mathrm{PEE}}$ alleles, respectively.

The strategy to genotype the Smad3 ${ }^{\text {null }}$ allele will be described elsewhere (N.R. Dunn and E.J. Robertson, in prep.).

\section{Mouse strains}

Smad2 ${ }^{\mathrm{CA} / \mathrm{CA}}, \operatorname{Smad2}{ }^{\mathrm{CA} / \mathrm{CA}} ;$ ROSA26 $^{\mathrm{R} / \mathrm{R}}$ (Soriano 1999), and Smad2 ${ }^{\mathrm{CA} / \mathrm{CA}^{\prime}}$;Smad3 ${ }^{\text {null/null }}$ (Datto et al. 1999) were maintained on a (129 X C57BL/6) hybrid background. Nodal ${ }^{413 . d /+}-129 /$ Sv//
Ev mice have been described (Conlon et al. 1994). Sox2Cre/ + Smad2 $2^{\mathrm{Robm} 1 /+}$ mice were obtained from crosses between Smad2 ${ }^{\text {Robm1/+ }}$ (Waldrip et al. 1998) and Sox2Cre/+ (Hayashi et al. 2002) parents and maintained on an outbred CD1 background. Mice carrying the $S m a d 2^{\mathrm{CD}}$ allele were generated by mating Smad2CA/CA animals with a Prm1-Cre deleter strain (O'Gorman et al. 1997) and were maintained on a CD1 background.

\section{Western blots}

Control extracts from KT15 Smad2-deficient ES cells (Tremblay et al. 2000) and COS cells transfected with human Smad2-Flag or Smad3-Flag expression constructs (kindly provided by C. Koonce) were prepared in $2 \times$ sample buffer. Yolk sac tissue from wild-type and Smad2 $\mathrm{CD} / \mathrm{CD}$ mutant embryos was sonicated in $2 \times$ sample buffer. Extracts were loaded on a 10\% SDS-PAGE gel. After transfer onto nitrocellulose membranes (Schleicher \& Schuell BioScience), the membranes were incubated with either a Smad2/3 antibody $(2 \mu \mathrm{g} / \mu \mathrm{L}$ mouse monoclonal antibody specific for the C-terminal part of the $\mathrm{MH1}$ and the linker domains, BD Transduction Laboratories, no. 610842) or a Smad4 antibody (1/1000 mouse monoclonal antibody, Santa Cruz Biotechnology, Inc., no. sc-7966). A horse radish peroxydase-conjugated sheep anti-mouse IgG (Amersham Pharmacia Biotech, no. NA931) was used as the secondary antibody. The blots were washed in TBST and developed by chemiluminescence using ECL (Amersham Pharmacia Biotech).

X-gal staining, whole-mount in situ hybridization, and histology

$\mathrm{X}$-gal staining and whole-mount in situ hybridization were performed according to standard procedures (Hogan et al. 1994). Probes for the following genes were used in this study: Gsc (Blum et al. 1992), Chordin (Klingensmith et al. 1999), Foxa2 (Sasaki and Hogan 1996), Nodal (Conlon et al. 1994), Shh (Echelard et al. 1993), T (Herrmann 1991), Cerl (Belo et al. 1997), Six3 (Oliver et al. 1995), Fgf8 (Crossley and Martin 1995), Hex (Thomas et al. 1998), Krox20 (Sham et al. 1993), and Otx2 (Ang et al. 1994). Noggin and $H n f 4 \alpha$ probe templates were generated by PCR. The Blimp-1 probe was derived from the EST mB3 (EST clone image: 1165721). For each probe and embryonic stage examined, between four and six mutant embryos were subject to whole-mount in situ hybridization and compared with a similar group of age-matched controls. For histology, embryos were fixed in $4 \%$ paraformaldehyde, dehydrated through an ethanol series, and embedded in wax before sectioning. Hematoxylin and eosin staining was performed according to standard protocols.

\section{Acknowledgments}

We are grateful to Liz Bikoff for discussions and for valuable comments on the manuscript. We thank Phil Soriano for generously providing the ROSA26 $6^{\mathrm{R}}$ mice, Josh Frederick and XiaFan Wang for generously providing the Smad3 ${ }^{\text {null }}$ mice, John Klingensmith for the Chordin probe, Chad Koonce for advice on Western analysis, Debbie Pelusi for genotyping assistance, and members of the lab for discussion. S.D.V. was supported by a Fellowship from the ARC and a Long Term Fellowship from the HFSP. N.R.D. was supported by a postdoctoral fellowship from the NICHD. This work was supported by a grant from the NIH to E.J.R.

The publication costs of this article were defrayed in part by 
payment of page charges. This article must therefore be hereby marked "advertisement" in accordance with 18 USC section 1734 solely to indicate this fact.

\section{References}

Agius, E., Oelgeschlager, M., Wessely, O., Kemp, C., and De Robertis, E.M. 2000. Endodermal Nodal-related signals and mesoderm induction in Xenopus. Development 127: 11731183.

Ang, S.L. and Rossant, J. 1994. HNF-3 $\beta$ is essential for node and notochord formation in mouse development. Cell 78: 561574.

Ang, S.L., Conlon, R.A., Jin, O., and Rossant, J. 1994. Positive and negative signals from mesoderm regulate the expression of mouse Otx2 in ectoderm explants. Development 120: 2979-2989.

Bachiller, D., Klingensmith, J., Kemp, C., Belo, J.A., Anderson, R.M., May, S.R., McMahon, J.A., McMahon, A.P., Harland, R.M., Rossant, J., et al. 2000. The organizer factors Chordin and Noggin are required for mouse forebrain development. Nature 403: 658-661.

Beddington, R.S. and Robertson, E.J. 1999. Axis development and early asymmetry in mammals. Cell 96: 195-209.

Belo, J.A., Bouwmeester, T., Leyns, L., Kertesz, N., Gallo, M., Follettie, M., and De Robertis, E.M. 1997. Cerberus-like is a secreted factor with neutralizing activity expressed in the anterior primitive endoderm of the mouse gastrula. Mech. Dev. 68: 45-57.

Blum, M., Gaunt, S.J., Cho, K.W., Steinbeisser, H., Blumberg, B., Bittner, D., and De Robertis, E.M. 1992. Gastrulation in the mouse: The role of the homeobox gene goosecoid. Cell 69: 1097-1106.

Brennan, J., Lu, C.C., Norris, D.P., Rodriguez, T.A., Beddington, R.S., and Robertson, E.J. 2001. Nodal signalling in the epiblast patterns the early mouse embryo. Nature 411: 965969.

Camus, A. and Tam, P.P. 1999. The organizer of the gastrulating mouse embryo. Curr. Top. Dev. Biol. 45: 117-153.

Conlon, F.L., Lyons, K.M., Takaesu, N., Barth, K.S., Kispert, A., Herrmann, B., and Robertson, E.J. 1994. A primary requirement for nodal in the formation and maintenance of the primitive streak in the mouse. Development 120: 19191928.

Crossley, P.H. and Martin, G.R. 1995. The mouse Fgf8 gene encodes a family of polypeptides and is expressed in regions that direct outgrowth and patterning in the developing embryo. Development 121: 439-451.

Datto, M.B., Frederick, J.P., Pan, L., Borton, A.J., Zhuang, Y., and Wang, X.F. 1999. Targeted disruption of Smad3 reveals an essential role in transforming growth factor $\beta$-mediated signal transduction. Mol. Cell. Biol. 19: 2495-2504.

David, N.B. and Rosa, F.M. 2001. Cell autonomous commitment to an endodermal fate and behaviour by activation of Nodal signalling. Development 128: 3937-3947.

Davidson, B.P., Kinder, S.J., Steiner, K., Schoenwolf, G.C., and Tam, P.P. 1999. Impact of node ablation on the morphogenesis of the body axis and the lateral asymmetry of the mouse embryo during early organogenesis. Dev. Biol. 211: 11-26.

de Souza, F.S., Gawantka, V., Gomez, A.P., Delius, H., Ang, S.L., and Niehrs, C. 1999. The zinc finger gene Xblimp1 controls anterior endomesodermal cell fate in Spemann's organizer. $E M B O$ T. 18: 6062-6072.

Dufort, D., Schwartz, L., Harpal, K., and Rossant, J. 1998. The transcription factor HNF3beta is required in visceral endo- derm for normal primitive streak morphogenesis. Development 125: 3015-3025.

Dyson, S. and Gurdon, J.B. 1998. The interpretation of position in a morphogen gradient as revealed by occupancy of activin receptors. Cell 93: 557-568.

Echelard, Y., Epstein, D.J., St-Jacques, B., Shen, L., Mohler, J., McMahon, J.A., and McMahon, A.P. 1993. Sonic hedgehog, a member of a family of putative signaling molecules, is implicated in the regulation of CNS polarity. Cell 75: 14171430.

Episkopou, V., Arkell, R., Timmons, P.M., Walsh, J.J., Andrew, R.L., and Swan, D. 2001. Induction of the mammalian node requires Arkadia function in the extraembryonic lineages. Nature 410: 825-830.

Gaudet, J. and Mango, S.E. 2002. Regulation of organogenesis by the Caenorhabditis elegans FoxA protein PHA-4. Science 295: 821-825.

Gritsman, K., Talbot, W.S., and Schier, A.F. 2000. Nodal signaling patterns the organizer. Development 127: 921-932.

Hallonet, M., Kaestner, K.H., Martin-Parras, L., Sasaki, H., Betz, U.A., and Ang, S.L. 2002. Maintenance of the specification of the anterior definitive endoderm and forebrain depends on the axial mesendoderm: A study using HNF3beta/Foxa2 conditional mutants. Dev. Biol. 243: 20-33.

Hayashi, S., Lewis, P., Pevny, L., and McMahon, A.P. 2002. Efficient gene modulation in mouse epiblast using a Sox2Cre transgenic mouse strain. Gene Expr. Patterns 2: 93-97.

Herrmann, B.G. 1991. Expression pattern of the Brachyury gene in whole-mount TWis/TWis mutant embryos. Development 113: 913-917.

Heyer, J., Escalante-Alcalde, D., Lia, M., Boettinger, E., Edelmann, W., Stewart, C.L., and Kucherlapati, R. 1999. Postgastrulation Smad2-deficient embryos show defects in embryo turning and anterior morphogenesis. Proc. Natl. Acad. Sci. 96: $12595-12600$.

Hogan, B.L., Beddington, R.S.,.Costantini, F., and Lacy, E. 1994. Manipulating the mouse embryo: A laboratory manual. Cold Spring Harbor Laboratory Press, Cold Spring Harbor, NY.

Hoodless, P.A., Pye, M., Chazaud, C., Labbe, E., Attisano, L., Rossant, J., and Wrana, J.L. 2001. FoxH1 (Fast) functions to specify the anterior primitive streak in the mouse. Genes \& Dev. 15: 1257-1271.

Howell, M. and Hill, C.S. 1997. XSmad2 directly activates the activin-inducible, dorsal mesoderm gene XFKH1 in Xenopus embryos. EMBO I. 16: 7411-7421.

Kimura, C., Yoshinaga, K., Tian, E., Suzuki, M., Aizawa, S., and Matsuo, I. 2000. Visceral endoderm mediates forebrain development by suppressing posteriorizing signals. Dev. Biol. 225: 304-321.

Klingensmith, J., Ang, S.L., Bachiller, D., and Rossant, J. 1999. Neural induction and patterning in the mouse in the absence of the node and its derivatives. Dev. Biol. 216: 535-549.

Lawson, K.A. 1999. Fate mapping the mouse embryo. Int. I. Dev. Biol. 43: 773-775.

Lowe, L.A., Yamada, S., and Kuehn, M.R. 2001. Genetic dissection of nodal function in patterning the mouse embryo. Development 128: 1831-1843.

Lu, C.C., Brennan, J., and Robertson, E.J. 2001. From fertilization to gastrulation: Axis formation in the mouse embryo. Curr. Opin. Genet. Dev. 11: 384-392.

Martinez Barbera, J.P., Clements, M., Thomas, P., Rodriguez, T., Meloy, D., Kioussis, D., and Beddington, R.S. 2000. The homeobox gene Hex is required in definitive endodermal tissues for normal forebrain, liver and thyroid formation. Development 127: 2433-2445. 
Massagué, J., Blain, S.W., and Lo, R.S. 2000. The TGFbeta signaling in growth control, cancer, and heritable disorders. Cell 103: 295-309.

Mukhopadhyay, M., Shtrom, S., Rodriguez-Esteban, C., Chen, L., Tsukui, T., Gomer, L., Dorward, D.W., Glinka, A., Grinberg, A., Huang, S.P., et al. 2001. Dickkopf1 is required for embryonic head induction and limb morphogenesis in the mouse. Dev. Cell 1: 423-434.

Norris, D.P. and Robertson, E.J. 1999. Asymmetric and nodespecific nodal expression patterns are controlled by two distinct cis-acting regulatory elements. Genes \& Dev. 13: $1575-1588$.

Norris, D.P., Brennan, J., Bikoff, E.K., and Robertson, E.J. 2002. The Foxh1-dependent autoregulatory enhancer controls the level of Nodal signals in the mouse embryo. Development 129: 3455-3468.

O'Gorman, S., Dagenais, N.A., Qian, M., and Marchuk, Y. 1997. Protamine-Cre recombinase transgenes efficiently recombine target sequences in the male germ line of mice, but not in embryonic stem cells. Proc. Natl. Acad. Sci. 94: 1460214607.

Oliver, G., Mailhos, A., Wehr, R., Copeland, N.G., Jenkins, N.A., and Gruss, P. 1995. Six3, a murine homologue of the sine oculis gene, demarcates the most anterior border of the developing neural plate and is expressed during eye development. Development 121: 4045-4055.

Ovchinnikov, D.A., Deng, J.M., Ogunrinu, G., and Behringer, R.R. 2000. Col2a1-directed expression of Cre recombinase in differentiating chondrocytes in transgenic mice. Genesis 26: $145-146$.

Perea-Gomez, A., Vella, F.D., Shawlot, W., Oulad-Abdelghani, M., Chazaud, C., Meno, C., Pfister, V., Chen, L., Robertson, E., Hamada, H., et al. 2002. Nodal antagonists in the anterior visceral endoderm prevent the formation of multiple primitive streaks. Dev. Cell 3: 745-756.

Rankin, C.T., Bunton, T., Lawler, A.M., and Lee, S.J. 2000. Regulation of left-right patterning in mice by growth/differentiation factor-1. Nat. Genet. 24: 262-265.

Rodriguez, T.A., Casey, E.S., Harland, R.M., Smith, J.C., and Beddington, R.S. 2001. Distinct enhancer elements control Hex expression during gastrulation and early organogenesis. Dev. Biol. 234: 304-316.

Saijoh, Y., Adachi, H., Sakuma, R., Yeo, C.Y., Yashiro, K., Watanabe, M., Hashiguchi, H., Mochida, K., Ohishi, S., Kawabata, M., et al. 2000. Left-right asymmetric expression of lefty 2 and nodal is induced by a signaling pathway that includes the transcription factor FAST2. Mol. Cell 5: 35-47.

Sasaki, H. and Hogan, B.L. 1996. Enhancer analysis of the mouse HNF-3 $\beta$ gene: Regulatory elements for node/notochord and floor plate are independent and consist of multiple sub-elements. Genes Cells 1: 59-72.

Sham, M.H., Vesque, C., Nonchev, S., Marshall, H., Frain, M., Gupta, R.D., Whiting, J., Wilkinson, D., Charnay, P., and Krumlauf, R. 1993. The zinc finger gene Krox20 regulates HoxB2 (Hox2.8) during hindbrain segmentation. Cell 72: 183-196.

Shawlot, W., Wakamiya, M., Kwan, K.M., Kania, A., Jessell, T.M., and Behringer, R.R. 1999. Lim1 is required in both primitive streak-derived tissues and visceral endoderm for head formation in the mouse. Development 126: 4925-4932.

Shiratori, H., Sakuma, R., Watanabe, M., Hashiguchi, H., Mochida, K., Sakai, Y., Nishino, J., Saijoh, Y., Whitman, M., and Hamada, H. 2001. Two-step regulation of left-right asymmetric expression of Pitx2: Initiation by nodal signaling and maintenance by Nkx2. Mol. Cell 7: 137-149.
Soriano, P. 1999. Generalized lacZ expression with the ROSA26 Cre reporter strain. Nat. Genet. 21: 70-71.

Stern, C.D. 2001. Initial patterning of the central nervous system: How many organizers? Nat. Rev. Neurosci. 2: 92-98.

Sulik, K., Dehart, D.B., Iangaki, T., Carson, J.L., Vrablic, T., Gesteland, K., and Schoenwolf, G.C. 1994. Morphogenesis of the murine node and notochordal plate. Dev. Dyn. 201: 260278.

Thomas, P. and Beddington, R. 1996. Anterior primitive endoderm may be responsible for patterning the anterior neural plate in the mouse embryo. Curr. Biol. 6: 1487-1496.

Thomas, P.Q., Brown, A., and Beddington., R.S 1998. Hex: A homeobox gene revealing peri-implantation asymmetry in the mouse embryo and an early transient marker of endothelial cell precursors. Development 125: 85-94.

Tremblay, K.D., Hoodless, P.A., Bikoff, E.K., and Robertson, E.J. 2000. Formation of the definitive endoderm in mouse is a Smad2-dependent process. Development 127: 3079-3090.

Varlet, I., Collignon, J., and Robertson, E.J.. 1997. nodal expression in the primitive endoderm is required for specification of the anterior axis during mouse gastrulation. Development 124: 1033-1044.

Vidal, F., Sage, J., Cuzin, F., and Rassoulzadegan, M. 1998. Cre expression in primary spermatocytes: A tool for genetic engineering of the germ line. Mol. Reprod. Dev. 51: 274-280.

Waldrip, W.R., Bikoff, E.K., Hoodless, P.A., Wrana, J.L., and Robertson, E.J. 1998. Smad2 signaling in extraembryonic tissues determines anterior-posterior polarity of the early mouse embryo. Cell 92: 797-808.

Wall, N.A., Craig, E.J., Labosky, P.A., and Kessler, D.S. 2000. Mesendoderm induction and reversal of left-right pattern by mouse Gdf1, a Vg1-related gene. Dev. Biol. 227: 495-509.

Weinstein, D.C., Ruiz i Altaba, A., Chen, W.S., Hoodless, P., Prezioso, V.R., Jessell, T.M., and Darnell Jr., J.E. 1994. The winged-helix transcription factor HNF-3 $\beta$ is required for notochord development in the mouse embryo. Cell 78: 575588.

Weisberg, E., Winnier, G.E., Chen, X., Farnsworth, C.L., Hogan, B.L., and Whitman, M. 1998. A mouse homologue of FAST-1 transduces TGF $\beta$ superfamily signals and is expressed during early embryogenesis. Mech. Dev. 79: 17-27.

Whitman, M. 2001. Nodal signaling in early vertebrate embryos. Themes and variations. Dev. Cell. 1: 605-617.

Yamamoto, M., Meno, C., Sakai, Y., Shiratori, H., Mochida, K., Ikawa, Y., Saijoh, Y., and Hamada, H. 2001. The transcription factor FoxH1 (FAST) mediates Nodal signaling during anterior-posterior patterning and node formation in the mouse. Genes \& Dev. 15: 1242-1256.

Zhou, X., Sasaki, H., Lowe, L., Hogan, B.L., and Kuehn, M.R. 1993. Nodal is a novel TGF- $\beta$-like gene expressed in the mouse node during gastrulation. Nature 361: 543-547. 


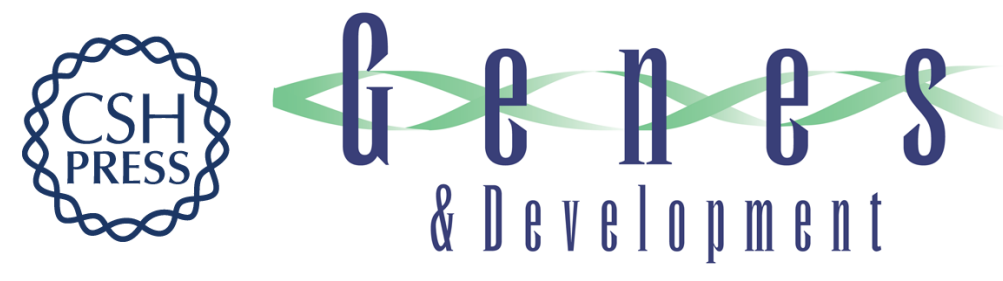

\section{Cell fate decisions within the mouse organizer are governed by graded Nodal signals}

Stéphane D. Vincent, N. Ray Dunn, Shigemi Hayashi, et al.

Genes Dev. 2003, 17:

Access the most recent version at doi:10.1101/gad.1100503

References This article cites 61 articles, 23 of which can be accessed free at: http://genesdev.cshlp.org/content/17/13/1646.full.html\#ref-list-1

License

Email Alerting

Receive free email alerts when new articles cite this article - sign up in the box at the top Service right corner of the article or click here.

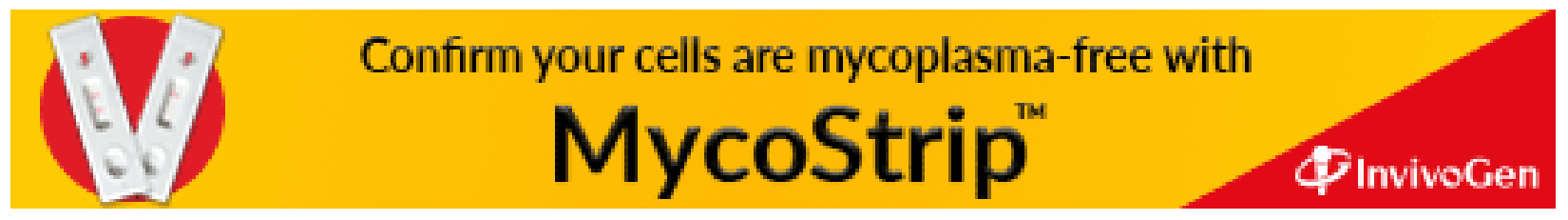

